\title{
Expanding the CRISPR Toolbox in Zebrafish for Studying Development and Disease
}

\author{
Kaili Liu, Cassidy Petree, Teresa Requena, Pratishtha Varshney and Gaurav K. Varshney*
}

Functional and Chemical Genomics Research Program, Oklahoma Medical Research Foundation, Oklahoma City, OK, United States

The study of model organisms has revolutionized our understanding of the mechanisms underlying normal development, adult homeostasis, and human disease. Much of what we know about gene function in model organisms (and its application to humans) has come from gene knockouts: the ability to show analogous phenotypes upon gene inactivation in animal models. The zebrafish (Danio rerio) has become a popular model organism for many reasons, including the fact that it is amenable to various forms of genetic manipulation. The RNA-guided CRISPR/Cas9-mediated targeted mutagenesis approaches have provided powerful tools to manipulate the genome toward developing new disease models and understanding the pathophysiology of

OPEN ACCESS

Edited by: Gokhan Dalgin, The University of Chicago, United States

Reviewed by: Mingyu Li,

Xiamen University, China Máté Varga,

Eötvös Loránd University, Hungary

*Correspondence:

Gaurav K. Varshney

gaurav-varshney@omrforg

Specialty section: This article was submitted to

Molecular Medicine,

a section of the journal

Frontiers in Cell and Developmental

Biology

Received: 22 November 2018 Accepted: 24 January 2019

Published: 04 March 2019

Citation:

Liu K, Petree C, Requena T, Varshney $P$ and Varshney GK (2019)

Expanding the CRISPR Toolbox in Zebrafish for Studying Development and Disease.

Front. Cell Dev. Biol. 7:13. doi: 10.3389/fcell.2019.00013 human diseases. CRISPR-based approaches are being used for the generation of both knockout and knock-in alleles, and also for applications including transcriptional modulation, epigenome editing, live imaging of the genome, and lineage tracing. Currently, substantial effort is being made to improve the specificity of Cas9, and to expand the target coverage of the Cas 9 enzymes. Novel types of naturally occurring CRISPR systems [Cas12a (Cpf1); engineered variants of Cas9, such as xCas9 and SpCas9-NG], are being studied and applied to genome editing. Since the majority of pathogenic mutations are single point mutations, development of base editors to convert C:G to T:A or A:T to G:C has further strengthened the CRISPR toolbox. In this review, we provide an overview of the increasing number of novel CRISPR-based tools and approaches, including lineage tracing and base editing.

Keywords: Cas12a (Cpf1), lineage tracing, base editors, zebrafish, disease models, CRISPR/Cas9

\section{INTRODUCTION}

Information gained from the study of model organisms is essential to our understanding of human development and disease. Replication of a mutant phenotype in a gene knockout (inactivation of a gene in an animal model) is considered to be the gold standard approach to support candidate gene predictions in humans. Zebrafish (Danio rerio) is uniquely suited to this approach and has become one of the fastest growing model organisms, useful for both basic and translational research (Bradford et al., 2017).

Zebrafish are an attractive alternative to mouse models because they give rise to a large number of progeny and are amenable to high-throughput mutagenesis and drug screening approaches (Kettleborough et al., 2013; Varshney et al., 2013; Varshney and Burgess, 2014; Gallardo et al., 2015). In addition, zebrafish fertilization is external, and their transparent larvae can be monitored throughout embryogenesis, providing unique accessibility to embryonic lethal mutations. 
The process of gene targeting in zebrafish is not as laborious as it is in mice, and the maintenance costs are 10X cheaper per animal (Varshney and Burgess, 2014). Importantly, zebrafish overcome an emerging technical issue in modeling disease pathology: many of the diseases studied today are multigenic, so disruption of a single gene may not produce a disease phenotype in any model system. However, combining genetic variants is straightforward in zebrafish, making it an ideal organism in which to model the functional consequences of multiple mutations. In addition, complementation studies in fish are relatively simple and allow for the direct testing of specific variants (not just knockouts) in a vertebrate system. The utility of zebrafish was further increased upon completion of the zebrafish genome-sequencing project (Howe et al., 2013); zebrafish and mammalian genes are highly conserved, and $70 \%$ of human disease genes have an ortholog in zebrafish (Howe et al., 2013). In zebrafish, many large-scale forward and reverse genetic screens have been performed using random mutagenesis methods - ENU or insertional mutagenesis (retroviral, Tol2, DS) - and the number of different genetic and molecular tools rapidly increased once the genome was sequenced (Amsterdam et al., 2011; Marquart et al., 2015; Quach et al., 2015; Seiler et al., 2015; Vrljicak et al., 2016). For decades, targeted gene knockouts were not possible in zebrafish, and its utility for validation studies of candidate genes was limited. This challenge was recently eliminated with the development of novel gene targeting approaches including ZFNs, TALENs, and CRISPR/Cas9 (Bedell et al., 2012; Jinek et al., 2012; Mali et al., 2013; Hsu et al., 2014; Varshney et al., 2015b); other techniques such as Structure Guided Nucleases (SGNs) have been shown to work for gene targeting in zebrafish but have not been adopted widely (Varshney and Burgess, 2016; Xu et al., 2016). With the transformative CRISPR/Cas9 approach, it is now possible to target any number of genes in an efficient and high-throughput manner (Gagnon et al., 2014; Shah et al., 2015; Varshney et al., 2015a). It is also possible to target multiple genes simultaneously; given that part of the zebrafish genome is duplicated, it is very useful in targeting 2 paralogs simultaneously (Jao et al., 2013).

CRISPR/Cas9 and other enzymes are not only being used to generate knockouts, introduce specific changes in the genome and repair mutant alleles, but are also being repurposed in other applications including transcriptional regulation, in vivo chromatin imaging, epigenome modulation, genome-wide knockout screens, etc. There are many reviews discussing the use of CRISPR-based approaches and the various technological developments in zebrafish (Varshney et al., 2015b; Li et al., 2016; Demarest and Brooks-Kayal, 2018). In this review we will focus on the latest development in expanding targeting coverage of gene targeting, base editing, transcriptional regulation, epigenome modulation, and lineage tracing.

\section{CRISPR-MEDIATED TARGETED MUTAGENESIS IN ZEBRAFISH}

In 2012, a joint team from Jennifer Doudna and Emmanuel Charpentier's lab, and an independent team from Virginijus
Siksnys's lab demonstrated that Cas9 from Streptococcus pyogenes or Streptococcus thermophilus together with CRISPR RNA (crRNA) can be guided to a target site to cleave DNA in vitro. Early the following year, George Church and Feng Zhang's labs utilized Cas9 from Streptococcus pyogenes and/or Streptococcus thermophilus to edit the genome in mammalian cells: they showed that single guide RNA (sgRNAs) can direct Cas9 to the target site to induce a double stranded break, which can then be repaired by either the non-homologous end joining (NHEJ) or homologydirected repair (HDR) pathways. An alternative repair pathway, microhomology-mediated end joining (aka Alt-EJ) (MMEJ; an error-prone repair mechanism that uses microhomologous sequences 5-25 bp in length) has also been shown to be activated by the double-stranded break induced by Cas9 (McVey and Lee, 2008; Ata et al., 2018). In the last 5-6 years, CRISPR-based genome editing tools have been used for many applications in a variety of cells, organisms and plants (Hsu et al., 2014). The use of simple and programmable CRISPR/Cas9 technology has completely transformed reverse genetics in zebrafish.

Zebrafish was the first vertebrate model used to demonstrate that CRISPR/Cas9 can efficiently edit the genome in vivo (Hwang et al., 2013) with up to 50\% targeting efficiency. Another report demonstrated that CRISPR/Cas9 can be used to generate biallelic mutations in gata5 and etsrp, and the observed phenotypes in injected embryos can phenocopy genetic mutants (Chang et al., 2013). Using a codon optimized version of Cas9 with nuclear localization signals, Jao et al. (2013) showed that Cas9 can efficiently induce biallelic mutations when Cas9 mRNA and sgRNA are injected into one-cell stage embryos. The authors further showed that up to five genes can be targeted simultaneously, and all showed phenotypes associated with each gene (Jao et al., 2013). It is evident from these initial reports that CRISPR/Cas9 is so efficient at inducing biallelic mutations that it allows for the generation of phenotypes in injected embryos similar to antisense morpholinos. Several strategies have been used to screen for phenotypes in the $\mathrm{F}_{0}$ generation in injected embryos; one such strategy used multiplexing to target multiple genes simultaneously and screen for phenotypes in $\mathrm{F}_{0}$. This approach was used to screen 48 genes and identify two novel genes involved in electrical-synapse formation (Shah et al., 2015). A similar strategy used a pool of four sgRNAs together with Cas9 protein to identify transcriptional regulators in cardiomyocytes; 50 candidate genes were screened and the role of $z b t b 16 a$ in cardiac development was identified (Wu et al., 2018). Burger et al. (2016) demonstrated that the use of in vitro assembled Cas9 mCherry or EGFP fusion protein, and sgRNA together as a ribonucleoprotein complex can provide a visual readout for efficient microinjections for the analysis of mutant phenotypes in $\mathrm{F}_{0}$ generation. These mutants were termed CRISPR-mediated mutants or crispants (akin to morphants; Burger et al., 2016). While these approaches to screen candidate genes by analyzing the expected phenotypes in injected embryos are efficient, in most cases a stable mutant is required for phenotypic analysis of gene function. Data from Shawn Burgess's lab targeting 89 genes show that genetic mutants can be generated with $\sim 28 \%$ germline transmission rates at a 99\% success rate. This high germline transmission rate is four-fivefold higher than 
that of other targeting approaches such as ZFNs, and TALENs (Varshney et al., 2015a).

Many groups have developed a streamlined workflow for generating mutants using CRISPR/Cas9 in a high-throughput manner (Gagnon et al., 2014; Varshney et al., 2015a, 2016a). The Burgess Lab addressed a few challenges in developing this workflow: First they developed a strategy to synthesize sgRNA by annealing two oligonucleotides that served as a template for in vitro transcription; this allowed for the synthesis of sgRNA in few hours with relatively low cost and is similar to the strategy was used by Gagnon et al. (2014). Secondly, the zebrafish genome is highly polymorphic, and it was predicted that this might cause multiple mismatches in the target sequence and prevent the sgRNA from binding efficiently. To address this, they sequenced the genome of the NHGRI-1 lab strain and identified more than 14 million variants. This data is available through UCSC genome browser track; while designing sgRNAs or PCR primers, variant regions of the genome can be avoided to maximize the success rate (LaFave et al., 2014). The third challenge they encountered was the identification of mutant alleles in a high-throughput manner. Several methods are currently used for the identification of mutants in zebrafish including DNA mismatch nuclease assays (Chang et al., 2013; Jao et al., 2013), restriction fragment length polymorphism (Hruscha et al., 2013) and sequencing (Gagnon et al., 2014; Varshney et al., 2015a; Burger et al., 2016), but none are amenable to high-throughput application. A method to determine the size of amplicons by fluorescent PCR was optimized to identify indels (Sood et al., 2013). This method uses three primers (gene-specific forward and reverse primers and a FAM-labeled primer) to amplify the regions around the target sites and resulting fluorescently labeled amplicons are mixed with a size standard (e.g., Rox400) to determine the amplicon size on ABI sequencing platform. This method can be applied in a highthroughput manner, and has resolution up to 1 bp (Figure 1) (Carrington et al., 2015; Varshney et al., 2015a).

Adopting CRISPR/Cas9 technology in a high-throughput manner for targeted mutagenesis has enabled geneticists to screen for large-numbers of genes with relatively modest resources, and generate disease models for ever-increasing candidate disease genes. The approaches have been widely applied: Pei et al. (2018) screened more than 200 candidate genes to identify genes involved in hair cell regeneration, and screens related to retinal regeneration or degeneration (Unal Eroglu et al., 2018) and several disease models including Niemman-Pick disease type C1;(Tseng et al., 2018), hearing disorders (Varshney et al., 2015a), congenital sideroblastic anemia with immunodeficiency, fevers and developmental delay (SIFD; (Giannelou et al., 2018), Mucolipidosis type IV (Li et al., 2017) have been performed. In zebrafish, phenotypes are generally studied in the $\mathrm{F}_{2}$ generation where homozygous embryos are generated by breeding two heterozygous $\left(F_{1}\right)$ lines over $\sim 6-7$ months. It has been shown that phenotypes can be screened in the $F_{1}$ generation in a nonMendelian manner by inbreeding two founders, thus eliminating a generation and saving time (Varshney et al., 2015a). This could be an important strategy that may speed up the phenotypic screening of a large number of candidate disease genes. To generate knockouts, Cas 9 is transiently expressed ubiquitously in one-cell stage embryos thus generating global knockouts, two independent studies have shown that Cas 9 can also be expressed in a tissue-specific manner thus it is possible to inactivate genes in a specific tissues (Ablain et al., 2015; Yin et al., 2015). Yin et al. (2015) demonstrated that by using a heat-shock inducible and tissue specific promoters, the expression of Cas 9 can be controlled both temporally and spatially [reviewed in (Li et al., 2016)]. They further characterized five U6 promoters to drive the expression of multiple guides thus adopting this approach for multiplex genome editing (Yin et al., 2015).

While CRISPR/Cas9 is an effective and simple tool for genetic manipulations, there are several concerns over its specificity as it has been shown to bind and edit unintended targets (e.g., Off targets) including inducing large deletions (Adikusuma et al., 2018). In zebrafish genetics, off-targets can easily be outcrossed away and a genotype-phenotype linkage must be established thus losing on-target activity by using Cas9 variants to achieve specificity should be considered. There are fewer studies in zebrafish that have tested the off-target effect in zebrafish. One such study detected off-target mutagenesis in only $1 / 25$ off target sites in germline, another study showed off-target mutation rates from 1.1 to $2.5 \%$ (Hruscha et al., 2013; Varshney et al., 2015a). Many Cas9 variants such as Cas9-HF1, eSpCas9, evoCas9, HypaCas9, and others have been developed to increase the specificity of the Cas 9 enzyme and thus reduce the off targets, however, these variant might also affect the on-target activities (Jamal et al., 2018).

We have summarized important CRISPR-based genome editing tools in Table 1. While SpCas9 can target multiple sites in the coding regions of the genome that is GC-rich, efforts are being made to expand target coverage by employing either orthologous Cas9 or evolving SpCas9 to identify different Protoacceptor Adjacent Motif (PAM) sequences.

\section{ENGINEERED AND NOVEL NUCLEASES TO EXPAND THE TARGETING COVERAGE}

Cas9 from Streptococcus pyogenes (spCas9) is the most popular and effective genome editing tool, and the sequences recognized by SpCas 9 are limited by the specific and simple PAM $\left(5^{\prime}\right.$-NGG-3') requirement (Jinek et al., 2012). However, this specific PAM sequence may not be available near the target of interest. To expand the targeting coverage, researchers have identified additional, naturally occurring CRISPR nucleases that may have different PAM requirements. Additionally, spCas9 was engineered to recognize other PAM sequences, expanding the targeting coverage and allowing them to be used in orthogonal applications. These newly identified CRISPR nucleases may also address the challenge of delivering the large size of spCas $9(1,368$ aa) as they may be smaller; they may also provide a homology template for in vivo therapeutic applications (Mout et al., 2017).

\section{Orthologous Cas9}

Many of the smaller-sized Cas9 nucleases discovered in different species can recognize different PAM sequences and varied lengths 
A

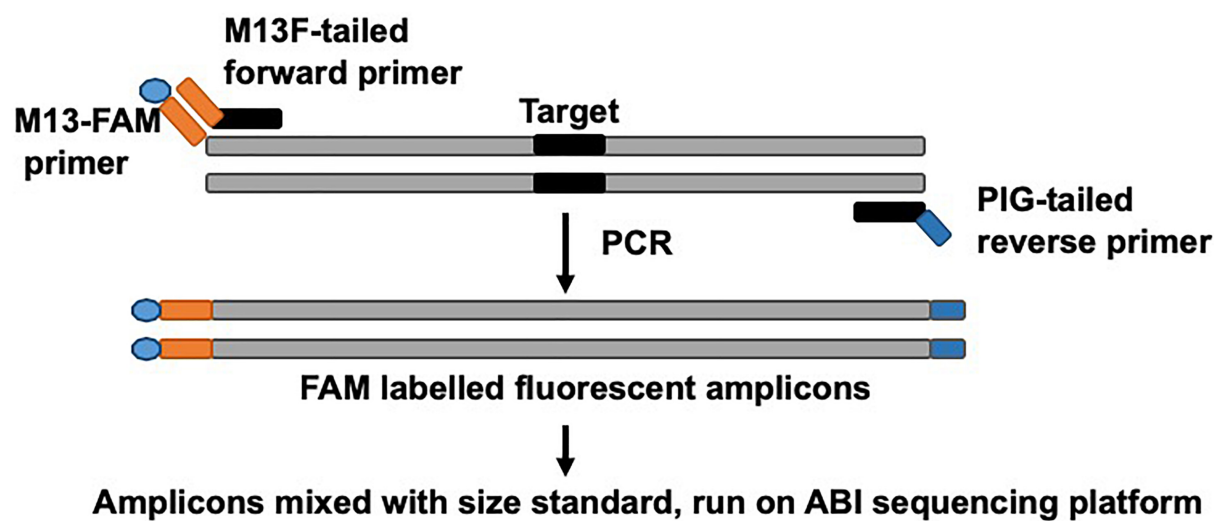

B

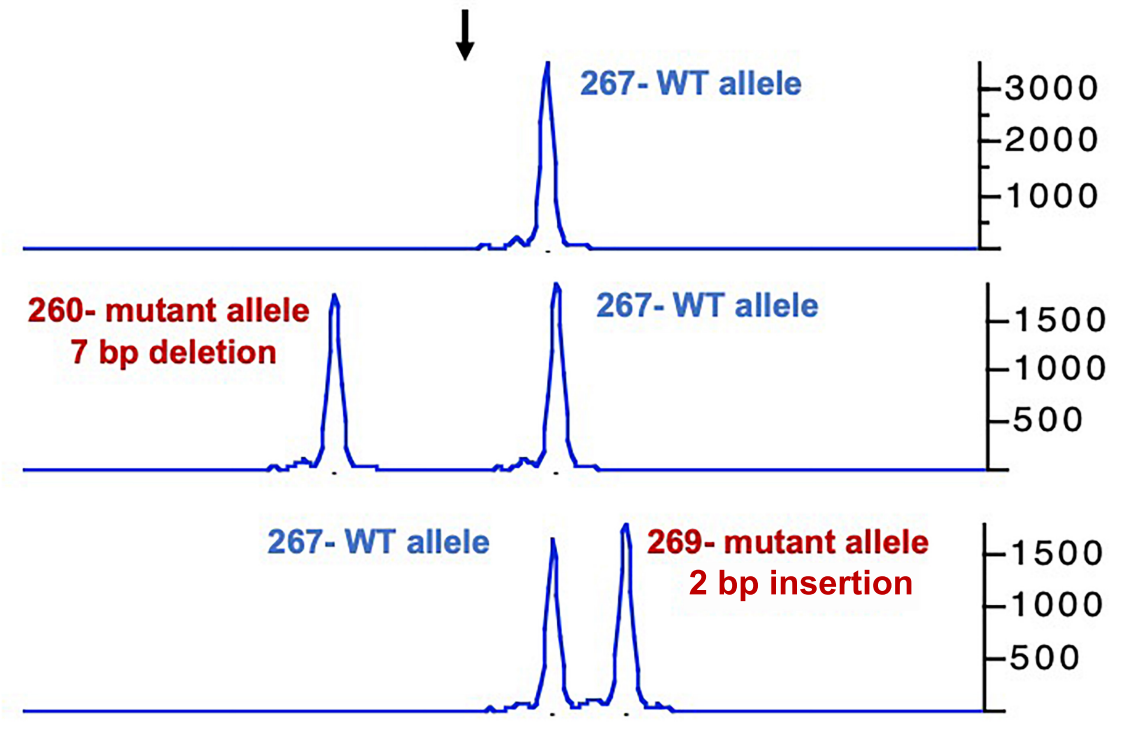

FIGURE 1 | Overview of mutant identification method using fragment analysis approach. (A) Gene specific primers are designed covering the target site (amplicon size ranging 200-300 bp). Gene-specific forward primer contains M13F sequence at the $5^{\prime}$, and reverse primer has PIG-tail sequence at $5^{\prime}$ end. PCR is performed using gene specific primer set, and a third primer with M13F sequence labeled with FAM, resulting amplicons are fluorescently labeled. (B) Fluorescently labeled primers are mixed with size standard (e.g., ROX-400), and run on ABI capillary sequencer, and data is analyzed using gene mapper software. The output will have the size of amplicon, wild type allele will have only one size, while mutant allele will have two different sizes. The indel size can be determined by comparing the size of two alleles (WT vs. mutant).

of target sequences for in vivo genome editing (Table 2): the Staphylococcus aureus Cas9 (SaCas9, $1053 \mathrm{aa}$ ) is not only small in size, but also uses a different complex PAM (NNGRRT; (Muller et al., 2016). Many other Cas9 nucleases from different bacterial species are being used for in vivo genome editing: Cas9 from Neisseria meningitidis (NmCas9) requires NNAGAAW PAM; Cas9 from Streptococcus thermophilus (St1Cas9, 1121 aa and St3Cas9, 1388 aa) require NNAGAAW and NGGNG PAMs, respectively; Cas9 nuclease from Campylobacter jejuni (CjCas9, $984 \mathrm{aa}$ ) recognizes a 22-nt target sequence with NNNVRYAC and NNNNRYAC PAM (Kim E. et al., 2017).

\section{Engineered Cas9 Variants}

Most of the orthologous Cas9 nucleases have long and complex PAM requirements that will limit the targeting range because they will occur less frequently in genomes. An alternative strategy to expand PAM specificity would be to engineer the
SpCas9 to recognize other PAMs. Kleinstiver et al. (2015b) engineered SpCas9 based on the crystal structure of the enzyme, and the mutated Cas9 was tested for its ability to recognize different PAM sites. Engineered SpCas9 variants VRER (D1135V/G1218R/R1335E/T1337R) recognizing NGCG PAM, VQR (D1135V/R1335Q/T1337R) recognizing NGAN or NGNG PAM, and EQR (D1135E/R1335Q/T1337R) variants recognizing NGAG PAM were generated. All of these SpCas9 variants were able to target sequences that were not targetable by wild-type SpCas9 in human cells, but only the VQR variant was able to target sites with NGAG PAMs (20-43\% efficiency in zebrafish; (Kleinstiver et al., 2015b). The efficiency of the VQR was further validated by showing its ability to target tyr and EGFP loci with 50 and 70\% efficiency, respectively. Zebrafish codon-optimized versions of VQR and EQR SpCas9 generated by Shawn Burgess' lab are also available from Addgene (Varshney et al., 2016a). 
TABLE 1 | Commonly used tools for CRISPR-mediated genome editing.

\begin{tabular}{|c|c|c|c|}
\hline Name & Description & URL & Reference \\
\hline CRISPRScan & Tool to design Cas9/Cas12a targets. & http://www.crisprscan.org & Moreno-Mateos et al., 2015 \\
\hline $\mathrm{CHOPCHOP}$ & $\begin{array}{l}\text { Tool to design Cas9, Cas9 variants, Cas12a targets, and genotyping } \\
\text { primers. A custom PAM can also be selected. }\end{array}$ & http://chopchop.cbu.uib.no & Labun et al., 2016 \\
\hline ccTop & Target prediction tool for multiple Cas9 and Cas12a. & https://crispr.cos.uni-heidelberg.de & Stemmer et al., 2017 \\
\hline Cas-Designer & $\begin{array}{l}\text { The most comprehensive tool to design Ca9, Cas9 variants, and } \\
\text { Cas } 12 \text { a targets. }\end{array}$ & http://www.rgenome.net/cas-designer & Park et al., 2015 \\
\hline MENTHU & $\begin{array}{l}\text { MENTHU (Microhomology-mediated End joining kNockout Target } \\
\text { Heuristic Utility) is a tool for designing targets with microhomologies, } \\
\text { to induce microhomology-mediated end-joining (MMEJ) deletions. }\end{array}$ & http://genesculpt.org/menthu & Ata et al., 2018 \\
\hline CRISPR-ERA & Cas9 target design tool for genome editing, repression, and activation & http://crispr-era.stanford.edu & Liu et al., 2015 \\
\hline CRISPResso 2 & $\begin{array}{l}\text { Webtool to analyze indels and base editing from the high-throughput } \\
\text { sequencing data }\end{array}$ & http://crispresso.pinellolab.partners.org/ & Clement et al., 2018 \\
\hline Cas-Analyzer & Online tool for analyzing indels from high-throughput sequencing data & http://www.rgenome.net/cas-analyzer/\#! & Park et al., 2017 \\
\hline CRISPR-GA & $\begin{array}{l}\text { CRISPR Genome Analyzer is a tool to identify indels from the } \\
\text { next-generation sequencing data }\end{array}$ & http://crispr-ga.net/ & Guell et al., 2014 \\
\hline CRISPRz & Database of validated sgRNA sequences in zebrafish & https://research.nhgri.nih.gov/CRISPRz/ & Varshney et al., 2016b \\
\hline inDelphi & $\begin{array}{l}\text { Tool to predicts the indels resulting from microhomology-mediated } \\
\text { end-joining (MMEJ) and non-homologous end-joining (NHEJ) repair. }\end{array}$ & https://www.crisprindelphi.design & Shen et al., 2018 \\
\hline FORECasT & Tool to predicts the indels generated by Cas9 & https://partslab.sanger.ac.uk/FORECasT & Allen et al., 2018 \\
\hline
\end{tabular}

As described above, the majority of the Cas9 orthologs or variants have complex PAM requirements, and the frequency of these targets in the genome is limited. To circumvent this challenge, David Liu's lab used phage-assisted continuous evolution (PACE) to isolate 14 evolved SpCas9 variants (xCas9 3.0-3.13); one such variant (xCas9 3.7) was able to recognize a broad range of PAM, including NG, NNG, CAA, GAT, and GAA (Hu et al., 2018). The xCas9 3.7 variant was able to cleave multiple PAMs at much higher frequency than wildtype SpCas9: GAA and GAT PAM showed $~ 5$-fold, NGT $\sim 4$.5-fold and NGC 2.1-fold efficiencies. Another variant, xCas9 3.6, showed the second-best editing efficiencies at fewer PAMs (Hu et al., 2018). SpCas9 was further engineered to generate a variant called SpCas9-NG that has a relaxed preference for the third nucleobases in the NGG PAM (Nishimasu et al., 2018). This variant had seven residues mutated (R1335V/L1111R/D1135V/G1218R/E1219F/A1322R/T1337R) in SpCas9; was capable of cleaving NGA, NGT, and NGG PAMs with more than $20 \%$ editing efficiency; and showed lower activity at NGC PAM. A comparison of editing efficiencies showed that spCas9-NG had higher editing efficiencies at NGA, NGT, and NGG sites, and xCas9 failed to edit NGC PAM targets (Nishimasu et al., 2018).

Similarly, Staphylococcus aureus Cas9 (SaCas9) was also modified using a molecular evolution strategy to recognize NNNRRT PAMs. This variant of SaCas9 is known as KKH SaCas9 (variant E782K/N968K, R1015H) and can further increase the SaCas9 targeting range by two-fourfold (Kleinstiver et al., 2015a). The KKH SaCas9 variant was able to recognize five independent targets in different genes with 10-90\% efficiency, thus expanding targeting coverage further in zebrafish (Feng et al., 2016).

The Cas9 nuclease from Francisella novocida (FnCas9) is one of the largest nucleases identified thus far (1629 aa) and recognizes NGG PAM similar to SpCas9, but has failed to generate indels in mammalian cells. It is possible that microinjecting mouse zygotes with FnCas9 protein and a guide RNA ribonucleoprotein complex may induce target-specific indels; a variant of FnCas9 (E1369R/E1449H/R1556A) called RHA FnCas9 could recognize YG PAM (Hirano et al., 2016).

Recently, a homolog of SpCas9 in Streptococcus macacae (SmacCas9) has been described to recognize the $5^{\prime}$-NAAN-3' PAM. A variant of SmacCas9 (iSpy-macCas9) was engineered to maintain its specificity for adenine dinucleotide PAM while showing higher genome editing efficiency in vivo (Jakimo et al., 2018). An orthologous Cas9 protein from Streptococcus canis (ScCas9) with 89.2\% sequence similarity to wild-type SpCas9 has also been characterized and shown to prefer a more minimal NNG (Chatterjee et al., 2018). An engineered version of ScCas9 ( $\triangle$ Loop $\triangle \mathrm{KQ}$ ) not only cleaves NGG PAM but also recognizes and edits NNGA PAM at a comparable rate, but it edits other NNGN PAMs with reduced efficiency. All of these engineered and orthologous Cas9 proteins have significantly expanded the targeted coverage.

\section{CRISPR/Cas12a (Cpf1)}

The majority of class 2 and type II nucleases and their engineered versions described earlier have preference for GC-rich PAMs that limits the targeting of AT-rich sequences, for example most of the non-coding genome in zebrafish is AT-rich (Howe et al., 2013). Another class 2 and type $\mathrm{V}$ family of nucleases, originally described as Cpf1 and later renamed Cas12a (Shmakov et al., 2017), was discovered as an alternative effective genome-editing tool (Zetsche et al., 2015). Cas12a is different from SpCas9 in many ways (Figure 2): (i) Cas12a recognizes T-rich PAM located at the $5^{\prime}$ end of the target DNA sequence, (ii) Cas12a is guided by a single CRISPR RNA (crRNA) that is shorter than that of SpCas9 and does not require trans-acting crRNA (tracrRNA), (iii) Cas12a 
TABLE 2 | Summary of Cas orthologs and variants.

\begin{tabular}{|c|c|c|c|c|}
\hline CRISPR Cas orthologs or variants & Recognized PAM & Target length & Use in zebrafish & Reference \\
\hline $\begin{array}{l}\text { Streptococcus pyogenes Cas9 } \\
\text { (SpCas9) }\end{array}$ & NGG & 19 or $20 \mathrm{nt}$ & Yes & $\begin{array}{l}\text { Jinek et al., 2012; Cong } \\
\text { et al., } 2013\end{array}$ \\
\hline $\begin{array}{l}\text { Streptococcus pyogenes Cas9 Variant } \\
\text { VQR (SpCas9 VQR) }\end{array}$ & NGAN, NGNG & 19 or $20 \mathrm{nt}$ & Yes & Kleinstiver et al., 2015b \\
\hline $\begin{array}{l}\text { Streptococcus pyogenes Cas9 Variant } \\
\text { EQR (SpCas9 EQR) }\end{array}$ & NGAG & 19 or $20 \mathrm{nt}$ & Yes & Kleinstiver et al., 2015b \\
\hline $\begin{array}{l}\text { Streptococcus pyogenes Cas9 Variant } \\
\text { VRER (SpCas9 VRER) }\end{array}$ & NGCG & $20 \mathrm{nt}$ & Yes & Kleinstiver et al., 2015b \\
\hline $\begin{array}{l}\text { Streptococcus pyogenes Cas9 Variant } \\
\text { D1135E (SpCas9 DE) }\end{array}$ & NAG & $20 \mathrm{nt}$ & Not tested & Kleinstiver et al., 2015b \\
\hline $\begin{array}{l}\text { Streptococcus pyogenes Cas9 Variant } \\
\text { QQR1 (SpCas9 QQR1) }\end{array}$ & NAAG & $20 \mathrm{nt}$ & Not tested & Anders et al., 2016 \\
\hline $\begin{array}{l}\text { Streptococcus pyogenes variant } \\
\text { TLIKDIV (xCas9 3.7) }\end{array}$ & $\begin{array}{l}\text { NG, NNG, CAA, GAT, } \\
\text { GAA }\end{array}$ & $20 \mathrm{nt}$ & Not tested & Hu et al., 2018 \\
\hline $\begin{array}{l}\text { Streptococcus pyogenes NG variant } \\
\text { (SpCas9NG) }\end{array}$ & NGA, NGT, NG & $20 \mathrm{nt}$ & Not tested & Nishimasu et al., 2018 \\
\hline Staphylococcus aureus Cas9 (SaCas9) & NNGRRT & $20-24 \mathrm{nt}$ & Not tested & Ran et al., 2015 \\
\hline $\begin{array}{l}\text { Staphylococcus aureus KKH Cas9 } \\
\text { variant (SaCas9 KKH) }\end{array}$ & NNNRRT & $21 \mathrm{nt}$ & Yes & Kleinstiver et al., 2015a \\
\hline $\begin{array}{l}\text { Streptococcus thermophilus } 1 \text { Cas9 } \\
\text { (St1Cas9) }\end{array}$ & NNAGAAW $(\mathrm{W}=\mathrm{A}$ or $\mathrm{T})$ & $20 \mathrm{nt}$ & Not tested & Muller et al., 2016 \\
\hline $\begin{array}{l}\text { Streptococcus thermophilus3 Cas9 } \\
\text { (St3Cas9) }\end{array}$ & NGGNG & $20 \mathrm{nt}$ & Not tested & Glemzaite et al., 2015 \\
\hline $\begin{array}{l}\text { Neisseria meningitidis Cas9 ( } \mathrm{Nm} \text { or } \\
\text { NmeCas9) }\end{array}$ & NNNNGMTT (M = A or C) & $23-24 \mathrm{nt}$ & Not tested & $\begin{array}{l}\text { Hou et al., 2013; Fonfara } \\
\text { et al., } 2014\end{array}$ \\
\hline Campylobacter jejuni Cas9 (CjCas9) & $\begin{array}{l}\text { NNNVRYAC NNNNRYAC } \\
\text { NNNVRYM ( } R=A \text { or } G) \\
(Y=C \text { or } T)(M=A \text { or } C)\end{array}$ & $22 \mathrm{nt}$ & Not tested & $\begin{array}{l}\text { Kim E. et al., 2017; Yamada } \\
\text { et al., } 2017\end{array}$ \\
\hline Francisella novicida Cas9 & $N G G$ & $22 \mathrm{nt}$ & Not tested & $\begin{array}{l}\text { Fonfara et al., 2014; Hirano } \\
\text { et al., } 2016\end{array}$ \\
\hline Francisella novicida Cas9 RHA variant & $Y G(Y=C$ or $T)$ & $22 \mathrm{nt}$ & Not tested & Hirano et al., 2016 \\
\hline Treponema denticola Cas9 (TdCas9) & NAAAAN & $20 \mathrm{nt}$ & Not tested & Esvelt et al., 2013 \\
\hline $\begin{array}{l}\text { Streptococcus macacae Cas9 } \\
\text { (SmacCas9) }\end{array}$ & NAAN & $20 \mathrm{nt}$ & Not tested & Jakimo et al., 2018 \\
\hline Streptococcus canis (ScCas9) & NNG & $20 \mathrm{nt}$ & Not tested & Chatterjee et al., 2018 \\
\hline $\begin{array}{l}\text { Streptococcus canis (ScCas9) } \\
\Delta L \text { oop } \Delta K Q \text { variant }\end{array}$ & NNGA, NGG & $20 \mathrm{nt}$ & Not tested & Chatterjee et al., 2018 \\
\hline $\begin{array}{l}\text { Acidaminococcus Cas12a } \\
\text { (AsCas12a/Cpf1) }\end{array}$ & $\pi T V(V=A$ or $C)$ & 23 or $24 \mathrm{nt}$ & Yes & Zetsche et al., 2015 \\
\hline $\begin{array}{l}\text { Lachnospiraceae Cas12a } \\
\text { (LbCas12a/Cpf1) }\end{array}$ & $\operatorname{TTV}(\mathrm{V}=\mathrm{A}$ or $\mathrm{C})$ & 23 or $24 \mathrm{nt}$ & Yes & Zetsche et al., 2015 \\
\hline Francisella Cas12a (FnCas12a/Cpf1) & $\begin{array}{l}T \mathrm{TN}, \mathrm{KYTV}(\mathrm{K}=\mathrm{G} \text { or } \mathrm{T}) \\
(\mathrm{Y}=\mathrm{C} \text { or } \mathrm{T})(\mathrm{V}=\mathrm{A} \text { or } \mathrm{C})\end{array}$ & 23 or $24 \mathrm{nt}$ & Yes & $\begin{array}{l}\text { Zetsche et al., 2015; Tu } \\
\text { et al., } 2017\end{array}$ \\
\hline Moraxella Cas12a (MbCas12a/Cpf1) & TTN & 23 or $24 \mathrm{nt}$ & Not tested & Zetsche et al., 2017 \\
\hline $\begin{array}{l}\text { AsCas12a, LbCas12a, FnCas12a, and } \\
\text { MbCas12a RR variants }\end{array}$ & $\begin{array}{l}\text { TYCV, TWTV }(\mathrm{W}=\mathrm{A} \text { or } \mathrm{T}) \\
(\mathrm{V}=\mathrm{A} \text { or } \mathrm{C})(\mathrm{Y}=\mathrm{C} \text { or } \mathrm{T})\end{array}$ & 23 or $24 \mathrm{nt}$ & Not tested & $\begin{array}{l}\text { Gao et al., 2017; Nishimasu } \\
\text { et al., 2017; Toth et al., } 2018\end{array}$ \\
\hline $\begin{array}{l}\text { AsCas12a, LbCas12a, FnCas12a, and } \\
\text { MbCas12a RVR variant }\end{array}$ & $\operatorname{TATV}(\mathrm{V}=\mathrm{A}$ or $\mathrm{C})$ & 23 or $24 \mathrm{nt}$ & Not tested & $\begin{array}{l}\text { Gao et al., 2017; Nishimasu } \\
\text { et al., 2017; Toth et al., } 2018\end{array}$ \\
\hline
\end{tabular}

uses an $\sim 23$ nt target sequence, (iv) Cas12a induces a doublestranded break in the target sequence via a staggered cut and $\sim 18$ nt distal to PAM, generating a 4-5 nt $5^{\prime}$ overhang, and (v) Cas $12 \mathrm{a}$ has both DNAse and RNase activity; therefore it is capable of processing its own CRISPR array (Fonfara et al., 2016). As of now, 32 Cas12a orthologs have been described, and their genomeediting potential was screened. Cas12a from Francisella novicida (FnCas12a), Acidaminococcus sp. BV3L6 (AsCas12a), and
Lachnospiraceae bacterial ND2006 (LbCas12a) exhibited robust editing in human cells, plants and many other model organisms, including zebrafish (Zetsche et al., 2015). Additionally, four other Cas12a orthologs [Thiomicrospira sp. Xs5 (TsCas12a), Moraxella bovoculi AAX08_00205 (Mb2Cas12a), Moraxella bovoculi AAX11_00205 (Mb3Cas12a), and Butyrivibrio sp. NC3005 (BsCas12a)] have been shown to induce indels in human cells, although only Mb3Cas12a was able to induce indels at a 


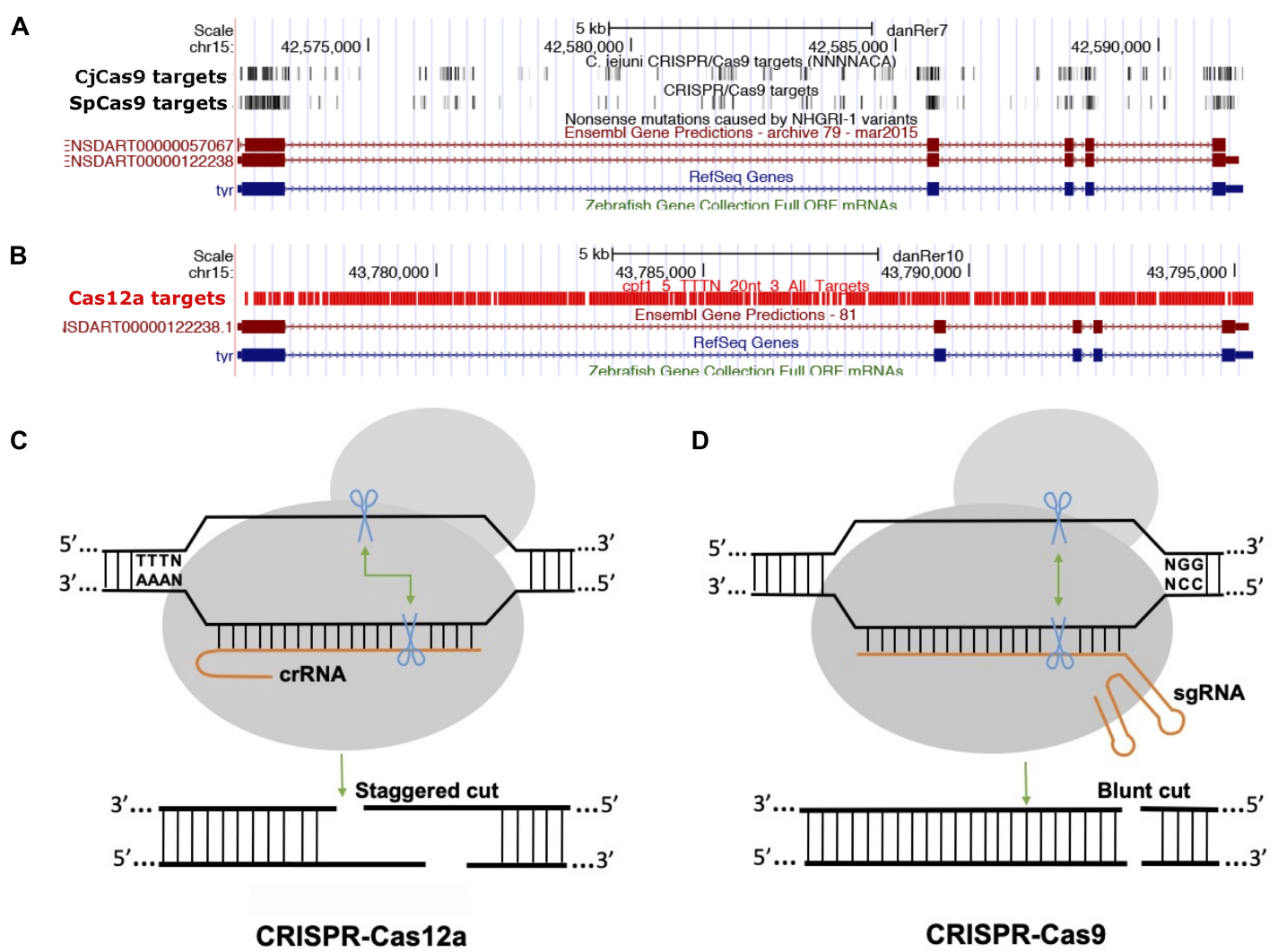

FIGURE 2 | Comparison between Cas9 and Cas12a. (A) Screenshot of UCSC genome browser tracks showing predicted target sequences in tyr gene for CjCas9, and SpCas9. The targets for both Cas9 nucleases are enriched in coding exons, (B) while Cas12 target sequences are enriched mostly in intronic sequences. (C,D) Comparison of Cas12a and Cas9, Cas12 a generates a staggered cut, Cas9 induces a blunt end cut.

rate comparable to AsCas12a and LbCas12a (Zetsche et al., 2017). AsCas12a and LbCas12a use TTTV PAM, while FnCas12a and Mb3Cas12a recognize the less-restrictive TTN and NTTN PAMs, respectively. FnCas12a also has been show to target sequences with KYTV PAM preference in mammalian cells (Tu et al., 2017). The Cas12a nucleases were further engineered by introducing mutations S542R/K607R and S542R/K548V/N552R to generate AsCas12aRR and AsCas12RVR variants, and G532R/K592R and G5323R/K538V/Y542R to generate LbCas12a RR or RVR variants, which can recognize non-canonical PAMs such as TYCV, TWTV, and TATV PAMs. Use of Cas12a in editing the zebrafish genome is not as straightforward as editing using SpCas9. Cas12a mRNA and crRNA targeting the tyr locus do not induce any indels in zebrafish at optimal temperature $\left(28^{\circ} \mathrm{C}\right)$ (Watkins-Chow et al., 2017). Further optimization revealed that Cas12a crRNAs are degraded rapidly after injection in onecell stage embryos; however, LbCas12a-crRNA ribonucleoprotein (RNP) complex can protect crRNA from degradation and can efficiently induce indels at a rate comparable to that of SpCas9 in zebrafish (Moreno-Mateos et al., 2017). LbCas12a is more effective in inducing indels than AsCas12a, and AsCas12a activity is temperature dependent in zebrafish. Heat shocking embryos after injection for $4 \mathrm{~h}$ at $34^{\circ} \mathrm{C}$ significantly increased the mutagenic activities for AsCas12a and LbCas12a nucleases.
(Moreno-Mateos et al., 2017) LbCas12a has been shown to achieve higher homology-directed repair compared to SpCas9. LbCas12a-mediated HDR is most efficient when an ssDNA donor template that is complementary to the target strand is provided (SpCas9 favors the non-target strand). Cas12a nucleases have expanded the targeted coverage many fold, which will help target non-coding regions that are AT rich in zebrafish (Moreno-Mateos et al., 2017).

\section{Base Editing Tools}

The majority of genetic diseases are caused by point mutations (single or multiple) that result in amino acid substitutions which generate non- or partially functional proteins. Studying these mutations in a model organism using gene knockout technology may not completely mimic the mutations found in human patients. Creating these mutations in zebrafish has been challenging: a targeted knock-in mutant is achieved via homologous recombination by delivering sgRNA and Cas9 together with either a single-stranded oligonucleotide or a donor plasmid containing the left and right homologous arms. Several strategies have been developed for introducing specific changes using knock-in technologies (Prykhozhij et al., 2018; Tessadori et al., 2018; Zhang et al., 2018), and reviewed in many publications (Albadri et al., 2017). 
However, the success rate of homology-directed repair (HDR) is extremely low, so introducing specific changes in the genome has been difficult because repair machinery tends to favor nonhomologous end joining repair. Moreover, HDR requires the delivery of donor template to the target cells and precise repair of the genomic sequence. Recently, Jeffery Essner's lab described an optimized targeted knock-in strategy, called GeneWeld, in which they developed a series of donor plasmids for gene tagging [pGTag-plasmids for Gene Tagging (58)]. This strategy is based on the targeting of multiple genomic loci using donor plasmids with short homology arms (24-48 bp), and can be used to integrate cargos up to $2 \mathrm{~kb}$ in zebrafish with high efficiency [up to $50 \%$ germline transmission (58)]. This technology should allow for maximal integration of fluorescent tags.

For introducing point mutations, recent progress in CRISPRmediated base editing allows for the introduction of point mutations (conversion of G-C base pairs to A-T base pairs or vice-versa) without inducing a double-stranded break (Figure 3; Komor et al., 2016; Gaudelli et al., 2017).

The first-generation CRISPR base editor (BE1) uses catalytically inactive Cas9 (dCas9) fused with cytidine deaminase enzyme encoded by the rat APOBEC1 gene (Komor et al., 2016). The cytidine deaminase enzyme converts cytosine bases into uridine, which are then read as thymine during replication. The result is a conversion of cytosine to thymine that occurs within the five-nucleotide window. The second-generation base editor (BE2) is fused with a uracil glycosylase inhibitor (UGI) that prevents excision of uracil during repair; BE2 has marginally higher activity compared to BE1 but does induce indel formation because it contains dCas9not. To further improve editing efficiency, the catalytic His residue at position 840 (which nicks the non-edited strand to mimic newly synthesized DNA) was restored to create $\mathrm{BE} 3$, the most widely used base editor. BE3 is the most efficient of the three base editors, and may also induce indels due to its nicking capabilities (Komor et al., 2016).
A new version of BE3 - HF-BE3 - was developed by incorporating mutations in Cas9 known to increase specificity and decrease off-target editing; in practice HF-BE3 appears to have lower on-target editing efficiency (Rees et al., 2017). Delivering the BE3- ribonucleoprotein complex (RNP) results in more robust editing than using plasmid-mediated delivery. This efficiency of BE3 RNP was further tested in zebrafish to generate specific point mutations targeting the tyrosinase locus: two of the three BE3:sgRNA RNP complexes were able to induce substantial point mutations in vivo, with 4-5\% editing efficiency (Rees et al., 2017).

David Liu's group continued their effort toward refining and improving the base editors. They engineered the next generation base editors (BE4) to increase the base conversion efficiency by $50 \%$. BE4 editors have extended (32 aa) rAPOBEC1-Cas9n and Cas9n-UGI linker (9 aa), and fusion of an additional UGI to the $\mathrm{C}$ terminus with another 9-amino acid linker. The BE4 base editor was further refined by adding the bacteriophage $\mathrm{Mu}$ protein Gam, which binds to double-stranded breaks and reduces indel formation to less than 1.5\%; this modified base editor is called BE4-Gam (Komor et al., 2017). To increase APOBEC1 expression, ancestral sequence reconstruction using 468 homologs of APOBEC1 was performed, and two ancestors (Anc689 and Anc687) were selected (Koblan et al., 2018). Codonoptimized bipartite NLS were added at the N- and C-termini, similar to BE4max, to create the AncBE4max variant that showed improved editing at multiple loci (Koblan et al., 2018).

In zebrafish, cytidine deaminase fused with Cas9 nickase was able to induce sequence-specific single base mutations from $\sim 9$ to $28 \%$ efficiency at multiple loci with a low number of indels (Zhang et al., 2017). Authors targeted tyr gene causal gene for human ocular albinism (OA) and oculocutaneous albinism (OCA). A mutation p.P301L in the tyr gene has been identified in OCA patients. While they were not able to convert proline to leucine, proline was converted to three other amino acids: serine, alanine or threonine. Edited embryos showed the loss

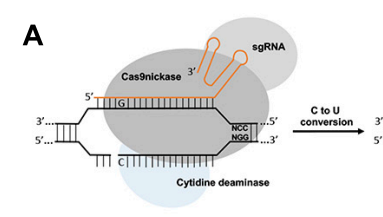

A

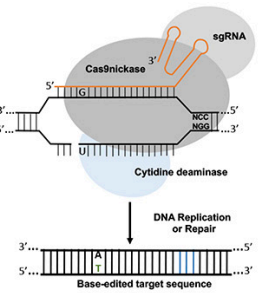

B

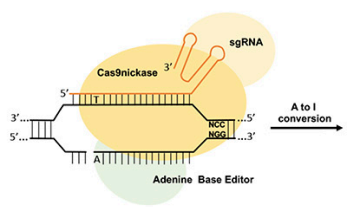

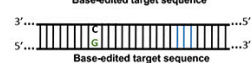

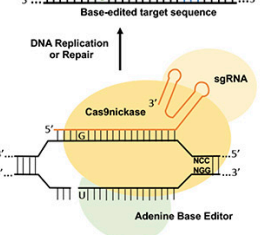

C

Cx35.4 GGTCATGgTTTATGTCGTAGCGg WT

CA G G G T C:A T G G T T TA TG T C GTA G C G G C A

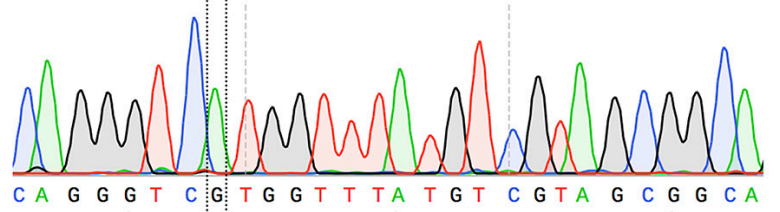

CA G G G T C G T TG G T T TA TGT C GTA G C G G CA

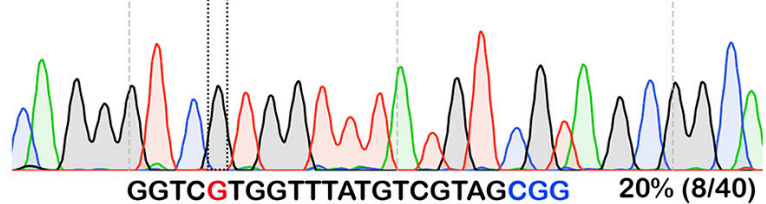

FIGURE 3 | Single nucleotide substitution using base editors. (A) Cytidine deaminase fused to nickase Cas9 converts cytosine to thymine to guanine to adenine within a targeting window. (B) Adenine base editor converts adenine to inosine that is recognized as guanine during DNA replication or repair thus converting $\mathrm{A}$ to $\mathrm{G}$ or C to T. (C) In vivo substitution of adenine to guanine using ABE7.10 base editor in zebrafish. sgRNA targeting cx35.4 gene was injected in 1-cell stage zebrafish embryos, DNA from a pool of four injected embryos was sequenced, and $20 \%$ of the clones carrying the desired $A$ to $G$ substitution. 
of pigmentation in the eyes of injected embryos. Five other targets tested also converted cytosine to thymine with varying efficiency (Zhang et al., 2017). While the BE system works in zebrafish, the efficiency is low compared to knockouts, and further optimization is required to improve editing efficiency.

Similar to the BE system, the "Target-AID system" was developed by Japanese researchers. The Target-AID system is composed of nuclease-dead Cas9 or Cas9 nickase fused with activation-induced cytidine deaminase (AID) encoded by the PmcDA1 gene from sea lamprey. Target-AID can also induce cytosine to thymine conversion within a five-nucleotide window. The target-AID system was used in zebrafish to introduce premature stop codons (TAG or TAA) by converting cytosine to thymine. Two genes, chordin (chd) and one-eyed pinhead (oep) were targeted using this strategy, and the introduction of premature stop codons phenocopies the known genetic mutants (Tanaka et al., 2018).

Recently, another base editor (eA3A-BE3) fused with an engineered human APOBEC3A (eA3A) domain was shown to deaminate cytidines in a more controlled manner, and function according to a TCR $>$ TCY $>$ VCN $(V=G, A, C, Y=C, T)$ hierarchy (Gehrke et al., 2018). The new base editor variant has shown comparable activities on cytidines in TC motifs, with reduced or no significant editing on cytidines in other motifs. Furthermore, eA3A-BE3 has shown low undesirable bystander mutations compared to other versions (Gehrke et al., 2018).

Existing cytosine deaminase base editors can target bases located between the 4th and 8th position in the target sequence. To expand the targeting window, a new base editor for programming larger $\mathrm{C}$ to $\underline{\mathrm{U}}(\mathrm{T})$ scope (BE-PLUS) was developed. This new editor utilizes the SunTag system (Jiang et al., 2018); SunTag contains multiple copies of GCN4 peptide (each consisting of 19 residues) which is recognized by a single chain variable (scFV) antibody. BE-PLUS contains three components: nickase Cas9 fused at the C-terminus to 10 copies of GCN4 peptide (SunTag), scFv-APOBEC-UGI-GB1, and sgRNA. BE-PLUS induced fewer C-T conversions at positions 4-8, but converted $\mathrm{C}-\mathrm{T}$ at $9-16$ positions more efficiently. However, at positions 4-8, BE-PLUS was as efficient as the previously described BE3 (Jiang et al., 2018).

Third- and fourth-generation base editors (BE3 and BE4Gam) were further optimized by codon optimizing Cas9, as well as by adding a FLAG tag and NLS at the N-terminus. These modified base editors were shown to improve C-T conversion up to 50-fold compared with the original BE3 or BE4 base editors (Zafra et al., 2018). A novel method - CRISPR-SKIP - has been shown to program exon skipping by mutating splice acceptor sites using cytidine deaminase (Gapinske et al., 2018). The CRISPRSKIP webtool can identify exons that can be skipped using this method, and it currently supports BE3, VQR-BE3, VRER-BE3, and SaKKH-BE3 variants (Gapinske et al., 2018). Michael Bassik's lab developed a novel base editor CRISPR-X, which uses an RNA aptamer (MS2) fused to sgRNA to recruit the cytidine deaminase to the target site and induce somatic hypermutation within a $100 \mathrm{bp}$ window. This is a powerful approach for protein engineering because it can generate a diverse population of alleles that could be useful for directed evolution (Hess et al., 2016).

\section{Adenine Base Editors}

Cytidine deaminase-based base editors convert C-T or G-A; there are no natural enzymes that can convert A-G or T-C. To address this problem, David Lius' lab developed an adenine base editor $(\mathrm{ABE})$ to modify adenine bases. The existing adenosine deaminase TadA/ADAR enzymes do not act on double-stranded DNA. Using phage-assisted continuous evolution (PACE), multiple rounds of directed evolution led to the identification of Escherichia coli TadA that can use DNA as a substrate. The ABE consists of a nickase Cas9 fused with a heterodimer of wild-type TadA and engineered TadA enzymes, guided by sgRNA to the target site. Engineered TadA converts adenine (A) to inosine (I) on the DNA target; inosine is recognized as guanine during DNA repair or replication, thus converting adenine (A) to guanine $(\mathrm{G})$ or thymine $(\mathrm{T})$ to cytosine $(\mathrm{C})$. Of the Liu lab's multiple versions from $\mathrm{ABE} 0.1$ to $\mathrm{ABE} 7, \mathrm{ABE7} .10$ has been shown to convert AT to GC with approximately $50 \%$ efficiency in mammalian cells. The ABE7.10 variant converts bases at position 4 to 7 , and ABE7.8 or ABE7.9 variants convert bases at positions 4-9. The ABE7.10 variant was further optimized to generate a new variant, ABEmax, by replacing SV40NLS to codon-optimized bipartite NLS at both the N- and C-termini. Modified ABEmax increases the base substitution rate from $\sim 1.5$ - to 2 -fold without changing the editing window; however, the rate of indels slightly increased.

Both cytidine deaminase and adenine deaminase enzymes are further fused with different variants such as VQR, VRER, SaCas9KKH, and newly evolved Cas9 such as xCas9, iSpymacCas9, and SpCas9-NG (Kim Y. B. et al., 2017; Hu et al., 2018). Thus, both types of base editors will provide coverage to change all four bases in a targeted manner. As the new variants and new orthologs of nucleases evolve the targeting coverage will further expand covering all of the pathogenic variants. Tables 3, 4 summarizes the different base editing resources, targeting range of each base editors mentioned above, respectively.

\section{TRANSCRIPTIONAL MODULATION AND EPIGENOME EDITING}

CRISPR/Cas9 has also been repurposed to modulate transcription and manipulate the epigenome. In order to apply the CRISPR system beyond inducing a double stranded break, the DNA cleavage activity of Cas9 nuclease must be inactivated. Cas9 from Streptococcus pyogenes (SpCas9) contains two nuclease domains - a RuvC-like domain and a $\mathrm{HNH}$ domain - both of which are required to induce a double stranded break (Jinek et al., 2012). Introducing mutations in the catalytic residues of both nuclease domains (D10A, H840A) will create a catalytically inactive version of the Cas9 (dCas9; Qi et al., 2013). Using sgRNA, dCas9 can be recruited to a specific target without inducing a DNA break. To modulate transcription, dCas9 was first fused with transcriptional activators (VP64, a synthetic tetramer of the Herpes Simplex Viral Protein or p65 a transcription factor activation domain) or transcriptional repressors [KRAB, a Kruppel-associated box and the transcriptional repressor of Koxl, or $4 \mathrm{X}$ mSin Interaction Domain (SID; Konermann et al., 2015)]. These fusion proteins 
TABLE 3 | Resources for base editing.

\begin{tabular}{|c|c|c|c|}
\hline Resource & Description & URL & Reference \\
\hline BE-Analyzer & NGS data analysis tool to identify based editing induced events. & http://www.rgenome.net/be-analyzer/ & Hwang et al., 2018 \\
\hline BE-Designer & Guide-RNA design tool for base editing. & http://www.rgenome.net/be-designer/ & Hwang et al., 2018 \\
\hline BEEP & $\begin{array}{l}\text { Command line program to assess CRISPR-mediated base editing } \\
\text { efficiency from Sanger sequencing ab1 files. }\end{array}$ & https://github.com/mitmedialab/BEEP & Chatterjee et al., 2018 \\
\hline CRISPR-SKIP & A tool to design induce exon skipping by base editing. & http://song.igb.illinois.edu/crispr-skip/ & Gapinske et al., 2018 \\
\hline CRISPResso 2 & Tool to analyze base editing events from next generation sequencing data. & http://crispresso.pinellolab.partners.org/ & Clement et al., 2018 \\
\hline EditR & Tool to estimate base editing by Sanger sequencing. & http://baseeditr.com/ & Kluesner et al., 2018 \\
\hline iSTOP & Database of sgRNAs for generating STOP codons using base editing. & http://www.ciccialab-database.com/istop & Billon et al., 2017 \\
\hline Beditor & Tool to design genome-wide sgRNA for base editing. & https://github.com/rraadd88/beditor & Dandage et al., 2018 \\
\hline
\end{tabular}

result in transcriptional activation (CRISPRa) or repression (CRISPRi) when targeted to the regulatory or coding regions of the gene.

Both CRISPRa and CRISPRi have been shown to work in modulating transcription of the target genes in zebrafish (Long et al., 2015). Two genes required for otic placode induction ( $f g f 8 a$ and foxil) were targeted to demonstrate the utility of CRISPRa and CRISPRi in zebrafish. When sgRNAs targeting $f g f 8 a$ coding

TABLE 4 | Summary of editing windows by base editors.

\begin{tabular}{|c|c|c|c|}
\hline Base editor (s) & $\begin{array}{l}\text { Editing } \\
\text { window }\end{array}$ & $\begin{array}{c}\text { Use in } \\
\text { zebrafish }\end{array}$ & Reference \\
\hline \multicolumn{4}{|l|}{ Cytosine deaminase } \\
\hline $\begin{array}{l}\text { SpCas9-BE1, SpCas9-BE2, } \\
\text { SpCas9-BE3, SpCas9-BE4 } \\
\text { SpCas9-BE4max, and } \\
\text { SpCas9-BE4-Gam }\end{array}$ & $4-8$ & Yes & $\begin{array}{l}\text { Komor et al., 2016, } \\
2017 \text {; Kim Y. B. et al., } \\
2017\end{array}$ \\
\hline SpCas9VQR-BE3 & $4-11$ & Yes & Kim Y. B. et al., 2017 \\
\hline SpCas9VRER-BE3 & $3-10$ & Not tested & Kim Y. B. et al., 2017 \\
\hline SpCas9YE1-BE3 & $4-7$ & Not tested & Kim Y. B. et al., 2017 \\
\hline $\begin{array}{l}\text { SpCas9YE2-BE3, } \\
\text { SpCas9YEE-BE3, } \\
\text { SpCas9YEE-BE3 }\end{array}$ & $5-6$ & Not tested & Kim Y. B. et al., 2017 \\
\hline $\begin{array}{l}\text { SaCas9-BE3, SaCas9-BE4, } \\
\text { SaCas9KKH-BE3 }\end{array}$ & $3-12$ & Not tested & Kim Y. B. et al., 2017 \\
\hline xCas9-BE3 & $4-8$ & Not tested & Hu et al., 2018 \\
\hline SpCas9 Target-AID & $2-4$ & Yes & Nishida et al., 2016 \\
\hline SpCas9-NG Target-AID & $2-4$ & Not tested & Nishimasu et al., 2018 \\
\hline SpCas9-BE-Plus & $4-16$ & Not tested & Jiang et al., 2018 \\
\hline $\begin{array}{l}\text { SpCas9 eA3A-BE3, } \\
\text { A3A-BE3 }\end{array}$ & $4-8$ & Not tested & Gehrke et al., 2018 \\
\hline CRISPR-X & $\begin{array}{l}-50 \text { bp to } \\
+50 \text { bp } \\
\text { relative to } \\
\text { PAM }\end{array}$ & Not tested & Hess et al., 2016 \\
\hline Cas12a (Cpf1)-BE & $8-13$ & Not tested & Li et al., 2018 \\
\hline \multicolumn{4}{|l|}{ Adenine base editors } \\
\hline ABE7.9 & $4-9$ & Not tested & Gaudelli et al., 2017 \\
\hline ABE7.10 & $4-8$ & Not tested & Gaudelli et al., 2017 \\
\hline xCas9 ABE & $4-8$ & Not tested & Hu et al., 2018 \\
\hline SpCas9-VQR ABE & $4-8$ & Not tested & Yang et al., 2018 \\
\hline SaCas9-KKH ABE & $6-12$ & Not tested & Yang et al., 2018 \\
\hline ScCas9-ABE7.10 & $4-8$ & Not tested & Chatterjee et al., 2018 \\
\hline
\end{tabular}

regions were co-injected with dCas9-KRAB (CRISPRi) fusion protein, the expression of $f g f 8 a$ was reduced at $11 \mathrm{hpf}$, and smaller otic vesicles were observed at $32 \mathrm{hpf}$ (Long et al., 2015). Similarly, when dCas9-VP160 (CRISPRa) together with either sgRNAs targeting $f g f 8 a$ or foxil were injected in one-cell stage embryos, the expression of $f g f 8 a$ and foxil was increased and the resulting animals showed enlarged otic vesicles.

The dCas9 was also fused with putative Eve repressor domain of zebrafish Evx1, and the resulting dCas9-Eve fusion together with three sgRNAs targeting sequences upstream of all zinc finger transcription factors $(z f n l 1 s)$ were used to inhibit the transcription of the znfl in zebrafish (Dong et al., 2017). Decreased expression of $z$ fll disrupts the formation of the posterior neuroectoderm in zebrafish gastrula, and the phenotype perfectly phenocopies that generated by the anti-sense morpholino (Dong et al., 2017).

In zebrafish it has been shown that mutants generated by targeting mutagenesis techniques, genetic compensation or transcriptional adaptation could all trigger the upregulation of related genes and compensate for the loss of the targeted gene. Such upregulation and compensation were not observed when antisense morpholinos were used, suggesting that downregulation of target genes using CRISPRi could be an alternate tool to study gene function.

Additionally, catalytically inactive Cas9 has been fused to various epigenetic effectors such as the catalytic core of the human acetyltransferase p300 which catalyzes acetylation of histone H3 lysine 27 (Hilton et al., 2015), histone demethylase (Kearns et al., 2015), histone deacetylase (HDAC) (Kwon et al., 2017) and many others (reviewed in Lau and Suh, 2018).

\section{LINEAGE TRACING USING CRISPR/Cas9}

A fundamental goal in developmental biology is to determine the origin of different cell types and tissues, and to establish their relationship in complex organisms. Lineage tracing is one method employed by developmental biologists to study the origin of cell types: techniques include dye based markers, nucleotide pulse-chase analysis, transplantation, sequencing somatic mutations, Cre-Lox and FLP-FRT based methods (reviewed in Kretzschmar and Watt, 2012). These methods can efficiently label cells at a single time-point to study large numbers of clonal populations in a complex animal, however, a detailed 
lineage tree over time cannot be reconstructed; understanding how cells change over the time will help determine the mechanisms of disease progression. Recently, the CRISPR/Cas9 mediated genome editing technique was used to generate genetic scars (indels) in the genome which serve as genetic barcodes for use in the reconstruction of cell lineages in developing animals or adults. Using this principle, many innovative approaches have been developed including genome editing of synthetic target arrays for lineage tracing (GESTALT; McKenna et al., 2016), lineage tracing by nuclease-activated editing of ubiquitous sequences (LINNAEUS; Spanjaard et al., 2018), ScarTrace (Alemany et al., 2018), and memory by engineered mutagenesis with optical in situ readout (MEMOIR; Frieda et al., 2017). CRISPR based lineage tracing is being adopted in multiple model organisms including zebrafish (Schmidt et al., 2017; Kalhor et al., 2018; Raj et al., 2018; Spanjaard et al., 2018). GESTALT, first applied to the understanding of the origin of organ development in zebrafish, was developed in the labs of Jay Shendure and Alex Schier. The Schier Lab engineered 10 different target sequences (unique barcodes in the $3^{\prime}$ UTR of DsRed) that are not found in the zebrafish genome to avoid any interreference with normal development. A transgenic line that drives the expression of DsRed under the ubiquitin promoter was generated. Ten sgRNAs that target the 10 unique sequences present in the transgenic lines were injected together with Cas 9 protein in one-cell stage zebrafish embryos. Embryos were collected at different time points, and target regions were amplified using primers containing unique molecular identifier (UMI) to add UMI barcodes in the amplicons. (The process of UMI tagging helps in assigning individual sequencing reads back to the cell of origin). Sequencing confirmed the in vivo allelic diversity, and the recovered alleles were used to reconstruct the lineage tree. To investigate whether these barcodes can also be recovered in adult animals, several organs (brain, eyes, intestine, gills, heart, and blood) were collected and subjected to DNA sequencing to recover barcode information. It was concluded that most cells in different adult organs were derived from fewer embryonic progenitors; more than 98\% of circulating blood in an adult zebrafish contains five common alleles, suggesting a highly clonal origin of the blood system in zebrafish. The GESTALT method was further modified by combining single-cell RNA sequencing to develop scGESTALT [single cell Genome Editing of Synthetic Target Arrays for Lineage Tracing; (Raj et al., 2018)]. The workflow for the cell lineage tracing and scRNA-seq experiment involves the introduction of sgRNAs to target exogenous sequences and the isolation of single cells at appropriate time points. This is followed by mRNA isolation, reverse transcription and cDNA amplification, library preparation, and sequencing both DNA and RNA; this method has been used to identify more than 100 different cell types during brain development. The scGESTALT method also allows barcodes to be recorded at post gastrulation stages by employing temporal control of Cas9 using a heat shock promoter and constitutively expressing sgRNA from the U6 promoter (Raj et al., 2018).

Unlike GESTALT, LINNAEUS, and ScarTrace approaches take advantage of existing transgenic lines carrying multiple integrations of a transgene - green fluorescent protein (GFP) or mCherry. Both LINNEAUS and ScarTrace combine lineage tracing with identification of cell types by singlecell transcriptomics. ScarTrace uses a zebrafish line carrying eight in-tandem copies of a histone-GFP transgene. sgRNA targeting the GFP with Cas9 protein is injected into one-cell stage embryos. Cas9 induces double-stranded breaks that when repaired by non-homologous end joining leave insertions or deletions (scars). During embryonic development, cells accumulate these scars and pass them on to future generations. When the scars are then sequenced, any adult cells containing identical scars must originate from a common progenitor cell. This method also defines cell types based on their transcriptome, thus cataloging both cell type and progenitor for each organ type. ScarTrace revealed that hematopoietic cells in the kidney marrow originated from fewer embryonic progenitors, and multiple progenitors give rise to specific cell types in the brain and eye. It was further revealed that a common progenitor produces both epidermal and mesenchymal cells of the caudal fin. Interestingly, this technique also showed how a progenitor cell commits to produce a left or right eye in zebrafish.

The LINNAEUS approach is similar to ScarTrace, however, LINNAEUS uses a zebrafish line carrying an RFP transgene with 16-32 independent integrations in the genome. The presence of independent integrations in different loci protect the scars from being removed or overwritten by Cas9. sgRNA targeting RFP and Cas9 were together injected into one-cell stage embryos; since RFP-targeting sgRNA generates indels (scars) and leave RFP non-functional, loss of RFP was used as a quality control to monitor the efficiency of editing and scar formation. At later time points, embryos were dissociated into single cells and RFP transcripts were sequenced to quantify the scar formation and the transcriptome from the same cell was sequenced by scRANAseq. Spanjaard and colleagues applied this approach to identify many different cell types from dissected adult organs including heart, liver, primary pancreatic islets and telencephalon. They found that immune cells from different organs can be grouped together in the lineage tree: analysis of cardiac and pancreatic cell types showed the early separation of myocardial and endocardial lineages.

MEMOIR method uses two different tools: Sequential single molecule Fluorescence In Situ Hybridization (smFISH) that reveals which specific genes are active in a particular cell, and CRISPR/Cas9 that generates indels (Frieda et al., 2017). MEMOIR uses bipartite genetic recording elements called barcoded scratchpads. Each scratchpad contains 10 repeat units, and sgRNAs and Cas 9 targeting scratchpads induces indels. There is also a barcode adjacent to each scratchpad which can be identified by smFISH and allows for the recording of each lineage. This method has so far only been used in vitro to record the cellular history of mouse embryonic stem cells through multiple generations (Frieda et al., 2017).

The methods described above generate complex lineage trees using scarring accumulated over several hours, and each method has a limited number of scars. To overcome this limitation, a selftargeting homing guide RNA (hgRNA) system was developed that can induce scarring over a longer time period and further 
increase complexity (Perli et al., 2016; Kalhor et al., 2018). Lineage tracing in mammals has been challenging compared to that in zebrafish. Kalhor et al. (2018) used hgRNAs to generate a mouse model for the study of cell lineages during early mouse development; hgRNA containing a targeting sequence with PAM was attached to a scaffold and allowed Cas9 to target the expression cassette. For in vivo cell lineage tracing, a transgenic mouse harboring 41 different homing guide RNA expression cassettes was created. This transgenic mouse was bred with a Cas9 expressing mouse strain to induce indels (barcodes). These barcodes can be used to track cells temporally and spatially (Kalhor et al., 2018).

These proof-of-principle studies have developed elegant lineage tracing strategies to establish lineage relationships and understand the fundamental mechanisms of cell differentiation under normal and pathological conditions (e.g., cancer metastasis) in complex model organisms including zebrafish. The development of CRISPR based lineage tracing methods is well-timed and complementary to the efforts toward building the human cell atlas (Regev et al., 2017). These approaches will have significant impact on our understanding of the origin of each cell type and how the adult body is developed from a single cell.

\section{CONCLUSION AND FUTURE PERSPECTIVES}

As described above the focus of recent research has been on developing strategies to improve Cas9 function, targeting coverage, and on-target efficiencies by reducing off target editing. CRISPR-based genome editing technologies have revolutionized biological research; CRISPR-related nucleases have been repurposed in many applications, and recent developments in

\section{REFERENCES}

Ablain, J., Durand, E. M., Yang, S., Zhou, Y., and Zon, L. I. (2015). A CRISPR/Cas9 vector system for tissue-specific gene disruption in zebrafish. Dev. Cell 32, 756-764. doi: 10.1016/j.devcel.2015.01.032

Adikusuma, F., Piltz, S., Corbett, M. A., Turvey, M., Mccoll, S. R., Helbig, K. J., et al. (2018). Large deletions induced by Cas9 cleavage. Nature 560, E8-E9. doi: 10.1038/s41586-018-0380-z

Albadri, S., Del Bene, F., and Revenu, C. (2017). Genome editing using CRISPR/Cas9-based knock-in approaches in zebrafish. Methods 12, 77-85. doi: 10.1016/j.ymeth.2017.03.005

Alemany, A., Florescu, M., Baron, C. S., Peterson-Maduro, J., and Van Oudenaarden, A. (2018). Whole-organism clone tracing using single-cell sequencing. Nature 556, 108-112. doi: 10.1038/nature25969

Allen, F., Crepaldi, L., Alsinet, C., Strong, A. J., Kleshchevnikov, V., De Angeli, P., et al. (2018). Predicting the mutations generated by repair of Cas9-induced double-strand breaks. Nat. Biotechnol. 37, 64-72. doi: 10.1038/nbt.4317

Amsterdam, A., Varshney, G. K., and Burgess, S. M. (2011). Retroviral-mediated insertional mutagenesis in zebrafish. Methods Cell Biol. 104, 59-82. doi: 10. 1016/B978-0-12-374814-0.00004-5

Anders, C., Bargsten, K., and Jinek, M. (2016). Structural plasticity of PAM recognition by engineered variants of the RNA-guided endonuclease Cas9. Mol. Cell 61, 895-902. doi: 10.1016/j.molcel.2016.02.020

Ata, H., Ekstrom, T. L., Martinez-Galvez, G., Mann, C. M., Dvornikov, A. V., Schaefbauer, K. J., et al. (2018). Robust activation of microhomology-mediated base editing and lineage tracing have further increased their utility in studying development and human diseases. New and inexpensive sequencing technologies are accelerating the discovery of candidate disease genes and pathogenic variants. CRISPR has provided a variety of tools to precisely modify the genome in a targeted manner for a variety of applications including functional gene knockouts, targeted induction or correction of single point mutations, and epigenome editing. Recent work has been focused on refining the specificity and expanding the target coverage of Cas9; directed evolution has led to the discovery of multiple Cas9 variants that will significantly expand the targeting coverage. Furthermore, development of base-editing techniques is an important milestone in the study of pathogenic variants in animal models; they will not only accelerate the functional validation of candidate disease genes in a model organism, but also accelerate the development of therapeutic tools for the treatment of a wide range of human diseases. Development of CRISPR-based lineage tracing methods are revealing information that could have been challenging to uncover using traditional approaches including the discovery of novel cell types and the origin of cells in different organs and tissues in complex model organisms.

\section{AUTHOR CONTRIBUTIONS}

KL, CP, TR, PV, and GV researched the data. GV wrote the article. All authors read the article and approved it for publication.

\section{FUNDING}

This research was supported by a grant from NIH/COBRE GM103636 (Project 3 to GV).

end joining for precision gene editing applications. PLoS Genet. 14:e1007652. doi: 10.1371/journal.pgen.1007652

Bedell, V. M., Wang, Y., Campbell, J. M., Poshusta, T. L., Starker, C. G., Krug, R. G., et al. (2012). In vivo genome editing using a high-efficiency TALEN system. Nature 491, 114-118. doi: 10.1038/nature11537

Billon, P., Bryant, E. E., Joseph, S. A., Nambiar, T. S., Hayward, S. B., Rothstein, R., et al. (2017). CRISPR-mediated base editing enables efficient disruption of eukaryotic genes through induction of STOP codons. Mol. Cell 67:e1064. doi: 10.1016/j.molcel.2017.08.008

Bradford, Y. M., Toro, S., Ramachandran, S., Ruzicka, L., Howe, D. G., Eagle, A., et al. (2017). Zebrafish models of human disease: gaining insight into human disease at ZFIN. ILAR J. 58, 4-16. doi: 10.1093/ilar/ ilw040

Burger, A., Lindsay, H., Felker, A., Hess, C., Anders, C., Chiavacci, E., et al. (2016). Maximizing mutagenesis with solubilized CRISPR-Cas9 ribonucleoprotein complexes. Development 143, 2025-2037. doi: 10.1242/dev. 134809

Carrington, B., Varshney, G. K., Burgess, S. M., and Sood, R. (2015). CRISPRSTAT: an easy and reliable PCR-based method to evaluate target-specific sgRNA activity. Nucleic Acids Res. 43:e157. doi: 10.1093/nar/gkv802

Chang, N., Sun, C., Gao, L., Zhu, D., Xu, X., Zhu, X., et al. (2013). Genome editing with RNA-guided Cas9 nuclease in zebrafish embryos. Cell Res. 23, 465-472. doi: $10.1038 /$ cr.2013.45

Chatterjee, P., Jakimo, N., and Jacobson, J. M. (2018). Minimal PAM specificity of a highly similar SpCas9 ortholog. Sci. Adv. 4:eaau0766. 
Clement, K., Rees, H., Canver, M., Gehrke, J., Farouni, R., Hsu, J., et al. (2018). Analysis and comparison of genome editing using CRISPResso2. bioRxiv [Preprint]. doi: 10.1101/392217

Cong, L., Ran, F. A., Cox, D., Lin, S., Barretto, R., Habib, N., et al. (2013). Multiplex genome engineering using CRISPR/Cas systems. Science 339, 819-823. doi: $10.1126 /$ science. 1231143

Dandage, R., Despres, P. C., Yachie, N., and Landry, C. R. (2018). beditor: A computational workflow for designing libraries of guide RNAs for CRISPRmediated base editing. bioRxiv [Preprint]. doi: 10.1101/426973

Demarest, S. T., and Brooks-Kayal, A. (2018). From molecules to medicines: the dawn of targeted therapies for genetic epilepsies. Nat. Rev. Neurol. 14, 735-745. doi: 10.1038/s41582-018-0099-3

Dong, X., Li, J., He, L., Gu, C., Jia, W., Yue, Y., et al. (2017). Zebrafish Znfl1 proteins control the expression of hoxblb gene in the posterior neuroectoderm by acting upstream of pou5f3 and sall4 genes. J. Biol. Chem. 292, 13045-13055. doi: 10.1074/jbc.M117.777094

Esvelt, K. M., Mali, P., Braff, J. L., Moosburner, M., Yaung, S. J., and Church, G. M. (2013). Orthogonal Cas9 proteins for RNA-guided gene regulation and editing. Nat. Methods 10, 1116-1121. doi: 10.1038/nmeth.2681

Feng, Y., Chen, C., Han, Y., Chen, Z., Lu, X., Liang, F., et al. (2016). Expanding CRISPR/Cas9 genome editing capacity in zebrafish using SaCas9. G3 (Bethesda) 6, 2517-2521. doi: 10.1534/g3.116.031914

Fonfara, I., Le Rhun, A., Chylinski, K., Makarova, K. S., Lecrivain, A. L., Bzdrenga, J., et al. (2014). Phylogeny of Cas9 determines functional exchangeability of dual-RNA and Cas9 among orthologous type II CRISPR-cas systems. Nucleic Acids Res. 42, 2577-2590. doi: 10.1093/nar/gkt1074

Fonfara, I., Richter, H., Bratoviè, M., Le Rhun, A., and Charpentier, E. (2016). The CRISPR-associated DNA-cleaving enzyme Cpf1 also processes precursor CRISPR RNA. Nature 532:517. doi: 10.1038/nature17945

Frieda, K. L., Linton, J. M., Hormoz, S., Choi, J., Chow, K. K., Singer, Z. S., et al. (2017). Synthetic recording and in situ readout of lineage information in single cells. Nature 541, 107-111. doi: 10.1038/nature20777

Gagnon, J. A., Valen, E., Thyme, S. B., Huang, P., Ahkmetova, L., Pauli, A., et al. (2014). Efficient mutagenesis by Cas9 protein-mediated oligonucleotide insertion and large-scale assessment of single-guide RNAs. PLoS One 9:e98186. doi: 10.1371/journal.pone.0098186

Gallardo, V. E., Varshney, G. K., Lee, M., Bupp, S., Xu, L., Shinn, P., et al. (2015). Phenotype-driven chemical screening in zebrafish for compounds that inhibit collective cell migration identifies multiple pathways potentially involved in metastatic invasion. Dis. Model Mech. 8, 565-576. doi: 10.1242/dmm.018689

Gao, L., Cox, D. B. T., Yan, W. X., Manteiga, J. C., Schneider, M. W., Yamano, T., et al. (2017). Engineered Cpf1 variants with altered PAM specificities. Nat. Biotechnol. 35, 789-792. doi: 10.1038/nbt.3900

Gapinske, M., Luu, A., Winter, J., Woods, W. S., Kostan, K. A., Shiva, N., et al. (2018). CRISPR-SKIP: programmable gene splicing with single base editors. Genome Biol. 19:107. doi: 10.1186/s13059-018-1482-5

Gaudelli, N. M., Komor, A. C., Rees, H. A., Packer, M. S., Badran, A. H., Bryson, D. I., et al. (2017). Programmable base editing of A · T to G · C in genomic DNA without DNA cleavage. Nature 551:464. doi: 10.1038/nature24644

Gehrke, J. M., Cervantes, O., Clement, M. K., Wu, Y., Zeng, J., Bauer, D. E., et al. (2018). An APOBEC3A-Cas9 base editor with minimized bystander and off-target activities. Nat. Biotechnol. 36, 977-982. doi: 10.1038/nbt.4199

Giannelou, A., Wang, H., Zhou, Q., Park, Y. H., Abu-Asab, M. S., Ylaya, K., et al. (2018). Aberrant tRNA processing causes an autoinflammatory syndrome responsive to TNF inhibitors. Ann. Rheum. Dis. 77, 612-619. doi: 10.1136/ annrheumdis-2017-212401

Glemzaite, M., Balciunaite, E., Karvelis, T., Gasiunas, G., Grusyte, M. M., Alzbutas, G., et al. (2015). Targeted gene editing by transfection of in vitro reconstituted Streptococcus thermophilus Cas9 nuclease complex. RNA Biol. 12, 1-4. doi: 10.1080/15476286.2015.1017209

Guell, M., Yang, L., and Church, G. M. (2014). Genome editing assessment using CRISPR genome analyzer (CRISPR-GA). Bioinformatics 30, 2968-2970. doi: 10.1093/bioinformatics/btu427

Hess, G. T., Fresard, L., Han, K., Lee, C. H., Li, A., Cimprich, K. A., et al. (2016). Directed evolution using dCas9-targeted somatic hypermutation in mammalian cells. Nat. Methods 13, 1036-1042. doi: 10.1038/nmeth.4038

Hilton, I. B., D’ippolito, A. M., Vockley, C. M., Thakore, P. I., Crawford, G. E., Reddy, T. E., et al. (2015). Epigenome editing by a CRISPR-Cas9-based acetyltransferase activates genes from promoters and enhancers. Nat. Biotechnol. 33, 510-517. doi: 10.1038/nbt.3199

Hirano, H., Gootenberg, J. S., Horii, T., Abudayyeh, O. O., Kimura, M., Hsu, P. D., et al. (2016). Structure and engineering of Francisella novicida Cas9. Cell 164, 950-961. doi: 10.1016/j.cell.2016.01.039

Hou, Z., Zhang, Y., Propson, N. E., Howden, S. E., Chu, L. F., Sontheimer, E. J., et al. (2013). Efficient genome engineering in human pluripotent stem cells using Cas9 from Neisseria meningitidis. Proc. Natl. Acad. Sci. U.S.A. 110, 15644-15649. doi: 10.1073/pnas.1313587110

Howe, K., Clark, M. D., Torroja, C. F., Torrance, J., Berthelot, C., Muffato, M., et al. (2013). The zebrafish reference genome sequence and its relationship to the human genome. Nature 496, 498-503. doi: 10.1038/nature12111

Hruscha, A., Krawitz, P., Rechenberg, A., Heinrich, V., Hecht, J., Haass, C., et al. (2013). Efficient CRISPR/Cas9 genome editing with low off-target effects in zebrafish. Development 140, 4982-4987. doi: 10.1242/dev.099085

Hsu, P. D., Lander, E. S., and Zhang, F. (2014). Development and applications of CRISPR-Cas9 for genome engineering. Cell 157, 1262-1278. doi: 10.1016/j.cell. 2014.05.010

Hu, J. H., Miller, S. M., Geurts, M. H., Tang, W., Chen, L., Sun, N., et al. (2018). Evolved Cas9 variants with broad PAM compatibility and high DNA specificity. Nature 556, 57-63. doi: 10.1038/nature26155

Hwang, G.-H., Park, J., Lim, K., Kim, S., Yu, J., Kim, S.-T., et al. (2018). Web-based design and analysis tools for CRISPR base editing. BMC Bioinformatics 19:542. doi: 10.1186/s12859-018-2585-4

Hwang, W. Y., Fu, Y., Reyon, D., Maeder, M. L., Tsai, S. Q., Sander, J. D., et al. (2013). Efficient genome editing in zebrafish using a CRISPR-Cas system. Nat. Biotechnol. 31, 227-229. doi: 10.1038/nbt.2501

Jakimo, N., Chatterjee, P., Nip, L., and Jacobson, J. M. (2018). A Cas9 with complete PAM recognition for adenine dinucleotides. bioRxiv [Preprint]. doi: 10.1101/ 429654

Jamal, M., Ullah, A., Ahsan, M., Tyagi, R., Habib, Z., and Rehman, K. (2018). Improving CRISPR-Cas9 on-target specificity. Curr. Issues Mol. Biol. 26, 65-80. doi: 10.21775/cimb.026.065

Jao, L. E., Wente, S. R., and Chen, W. (2013). Efficient multiplex biallelic zebrafish genome editing using a CRISPR nuclease system. Proc. Natl. Acad. Sci. U.S.A. 110, 13904-13909. doi: 10.1073/pnas.1308335110

Jiang, W., Feng, S., Huang, S., Yu, W., Li, G., Yang, G., et al. (2018). BE-PLUS: a new base editing tool with broadened editing window and enhanced fidelity. Cell Res. 28, 855-861. doi: 10.1038/s41422-018-0052-4

Jinek, M., Chylinski, K., Fonfara, I., Hauer, M., Doudna, J. A., and Charpentier, E. (2012). A programmable dual-RNA-guided DNA endonuclease in adaptive bacterial immunity. Science 337, 816-821. doi: 10.1126/science.1225829

Kalhor, R., Kalhor, K., Mejia, L., Leeper, K., Graveline, A., Mali, P., et al. (2018). Developmental barcoding of whole mouse via homing CRISPR. Science 361:eaat9804.

Kearns, N. A., Pham, H., Tabak, B., Genga, R. M., Silverstein, N. J., Garber, M., et al. (2015). Functional annotation of native enhancers with a Cas9-histone demethylase fusion. Nat. Methods 12, 401-403. doi: 10.1038/nmeth.3325

Kettleborough, R. N., Busch-Nentwich, E. M., Harvey, S. A., Dooley, C. M., De Bruijn, E., Van Eeden, F., et al. (2013). A systematic genome-wide analysis of zebrafish protein-coding gene function. Nature 496, 494-497. doi: 10.1038/ nature 11992

Kim, E., Koo, T., Park, S. W., Kim, D., Kim, K., Cho, H. Y., et al. (2017). In vivo genome editing with a small Cas9 orthologue derived from Campylobacter jejuni. Nat. Commun. 8:14500. doi: 10.1038/ncomms 14500

Kim, Y. B., Komor, A. C., Levy, J. M., Packer, M. S., Zhao, K. T., and Liu, D. R. (2017). Increasing the genome-targeting scope and precision of base editing with engineered Cas9-cytidine deaminase fusions. Nat. Biotechnol. 35, 371-376. doi: $10.1038 /$ nbt. 3803

Kleinstiver, B. P., Prew, M. S., Tsai, S. Q., Nguyen, N. T., Topkar, V. V., Zheng, Z., et al. (2015a). Broadening the targeting range of Staphylococcus aureus CRISPRCas9 by modifying PAM recognition. Nat. Biotechnol. 33, 1293-1298. doi: 10. 1038/nbt.3404

Kleinstiver, B. P., Prew, M. S., Tsai, S. Q., Topkar, V. V., Nguyen, N. T., Zheng, Z., et al. (2015b). Engineered CRISPR-Cas9 nucleases with altered PAM specificities. Nature 523, 481-485. doi: 10.1038/nature14592

Kluesner, M. G., Nedveck, D. A., Lahr, W. S., Garbe, J. R., Abrahante, J. E., Webber, B. R., et al. (2018). EditR: A Method to Quantify Base 
Editing from Sanger Sequencing. CRISPR J. 1, 239-250. doi: 10.1089/crispr. 2018.0014

Koblan, L. W., Doman, J. L., Wilson, C., Levy, J. M., Tay, T., Newby, G. A., et al. (2018). Improving cytidine and adenine base editors by expression optimization and ancestral reconstruction. Nat. Biotechnol. 36, 843-846. doi: 10.1038/nbt. 4172

Komor, A. C., Kim, Y. B., Packer, M. S., Zuris, J. A., and Liu, D. R. (2016). Programmable editing of a target base in genomic DNA without doublestranded DNA cleavage. Nature 533, 420-424. doi: 10.1038/nature17946

Komor, A. C., Zhao, K. T., Packer, M. S., Gaudelli, N. M., Waterbury, A. L., Koblan, L. W., et al. (2017). Improved base excision repair inhibition and bacteriophage Mu Gam protein yields C:G-to-T:A base editors with higher efficiency and product purity. Sci. Adv. 3:eaao4774.

Konermann, S., Brigham, M. D., Trevino, A. E., Joung, J., Abudayyeh, O. O., Barcena, C., et al. (2015). Genome-scale transcriptional activation by an engineered CRISPR-Cas9 complex. Nature 517, 583-588. doi: 10.1038/ nature 14136

Kretzschmar, K., and Watt, F. M. (2012). Lineage tracing. Cell 148, 33-45. doi: 10.1016/j.cell.2012.01.002

Kwon, D. Y., Zhao, Y. T., Lamonica, J. M., and Zhou, Z. (2017). Locusspecific histone deacetylation using a synthetic CRISPR-Cas9-based HDAC. Nat. Commun. 8:15315. doi: 10.1038/ncomms 15315

Labun, K., Montague, T. G., Gagnon, J. A., Thyme, S. B., and Valen, E. (2016). CHOPCHOP v2: a web tool for the next generation of CRISPR genome engineering. Nucleic Acids Res. 44, W272-W276. doi: 10.1093/nar/gkw398

LaFave, M. C., Varshney, G. K., Vemulapalli, M., Mullikin, J. C., and Burgess, S. M. (2014). A defined zebrafish line for high-throughput genetics and genomics: NHGRI-1. Genetics 198, 167-170. doi: 10.1534/genetics.114.166769

Lau, C. H., and Suh, Y. (2018). In vivo epigenome editing and transcriptional modulation using CRISPR technology. Transgenic Res. 27, 489-509. doi: 10. 1007/s11248-018-0096-8

Li, H., Pei, W., Vergarajauregui, S., Zerfas, P. M., Raben, N., Burgess, S. M., et al. (2017). Novel degenerative and developmental defects in a zebrafish model of mucolipidosis type IV. Hum. Mol. Genet. 26, 2701-2718. doi: 10.1093/hmg/ ddx 158

Li, M., Zhao, L., Page-Mccaw, P. S., and Chen, W. (2016). Zebrafish genome engineering using the CRISPR-Cas9 system. Trends Genet. 32, 815-827. doi: 10.1016/j.tig.2016.10.005

Li, X., Wang, Y., Liu, Y., Yang, B., Wang, X., Wei, J., et al. (2018). Base editing with a Cpf1-cytidine deaminase fusion. Nat. Biotechnol. 36, 324-327. doi: 10.1038/ nbt. 4102

Liu, H., Wei, Z., Dominguez, A., Li, Y., Wang, X., and Qi, L. S. (2015). CRISPR-ERA: a comprehensive design tool for CRISPR-mediated gene editing, repression and activation. Bioinformatics 31, 3676-3678. doi: 10.1093/ bioinformatics/btv 423

Long, L., Guo, H., Yao, D., Xiong, K., Li, Y., Liu, P., et al. (2015). Regulation of transcriptionally active genes via the catalytically inactive Cas9 in C. elegans and D. rerio. Cell Res. 25, 638-641. doi: 10.1038/cr.2015.35

Mali, P., Yang, L., Esvelt, K. M., Aach, J., Guell, M., Dicarlo, J. E., et al. (2013). RNA-guided human genome engineering via Cas9. Science 339, 823-826. doi: $10.1126 /$ science. 1232033

Marquart, G. D., Tabor, K. M., Brown, M., Strykowski, J. L., Varshney, G. K., Lafave, M. C., et al. (2015). A 3D searchable database of transgenic zebrafish Gal4 and Cre lines for functional neuroanatomy studies. Front. Neural Circ. 9:78. doi: $10.3389 /$ fncir.2015.00078

McKenna, A., Findlay, G. M., Gagnon, J. A., Horwitz, M. S., Schier, A. F., and Shendure, J. (2016). Whole-organism lineage tracing by combinatorial and cumulative genome editing. Science 353:aaf7907. doi: 10.1126/science. aaf7907

McVey, M., and Lee, S. E. (2008). MMEJ repair of double-strand breaks (director's cut): deleted sequences and alternative endings. Trends Genet. 24, 529-538. doi: 10.1016/j.tig.2008.08.007

Moreno-Mateos, M. A., Fernandez, J. P., Rouet, R., Vejnar, C. E., Lane, M. A., Mis, E., et al. (2017). CRISPR-Cpfl mediates efficient homology-directed repair and temperature-controlled genome editing. Nat. Commun. 8:2024. doi: 10. 1038/s41467-017-01836-2

Moreno-Mateos, M. A., Vejnar, C. E., Beaudoin, J. D., Fernandez, J. P., Mis, E. K., Khokha, M. K., et al. (2015). CRISPRscan: designing highly efficient sgRNAs for
CRISPR-Cas9 targeting in vivo. Nat. Methods 12, 982-988. doi: 10.1038/nmeth. 3543

Mout, R., Ray, M., Lee, Y. W., Scaletti, F., and Rotello, V. M. (2017). In Vivo delivery of CRISPR/Cas9 for therapeutic gene editing: progress and challenges. Bioconjug Chem. 28, 880-884. doi: 10.1021/acs.bioconjchem.7b00057

Muller, M., Lee, C. M., Gasiunas, G., Davis, T. H., Cradick, T. J., Siksnys, V., et al. (2016). Streptococcus thermophilus CRISPR-Cas9 systems enable specific editing of the human genome. Mol. Ther. 24, 636-644. doi: 10.1038/mt.2015.218

Nishida, K., Arazoe, T., Yachie, N., Banno, S., Kakimoto, M., Tabata, M., et al. (2016). Targeted nucleotide editing using hybrid prokaryotic and vertebrate adaptive immune systems. Science 353:aaf8729. doi: 10.1126/science. aaf 8729

Nishimasu, H., Shi, X., Ishiguro, S., Gao, L., Hirano, S., Okazaki, S., et al. (2018). Engineered CRISPR-Cas9 nuclease with expanded targeting space. Science 361, 1259-1262. doi: 10.1126/science.aas9129

Nishimasu, H., Yamano, T., Gao, L., Zhang, F., Ishitani, R., and Nureki, O. (2017). Structural basis for the altered PAM recognition by engineered CRISPR-Cpf1. Mol. Cell 67:e132. doi: 10.1016/j.molcel.2017.04.019

Park, J., Bae, S., and Kim, J. S. (2015). Cas-designer: a web-based tool for choice of CRISPR-Cas9 target sites. Bioinformatics 31, 4014-4016. doi: 10.1093/ bioinformatics/btv537

Park, J., Lim, K., Kim, J. S., and Bae, S. (2017). Cas-analyzer: an online tool for assessing genome editing results using NGS data. Bioinformatics 33, 286-288. doi: 10.1093/bioinformatics/btw561

Pei, W., Xu, L., Huang, S. C., Pettie, K., Idol, J., Rissone, A., et al. (2018). Guided genetic screen to identify genes essential in the regeneration of hair cells and other tissues. NPJ Regen. Med. 3:11. doi: 10.1038/s41536-018-0050-7

Perli, S. D., Cui, C. H., and Lu, T. K. (2016). Continuous genetic recording with self-targeting CRISPR-Cas in human cells. Science 353:aag0511. doi: 10.1126/ science.aag0511

Prykhozhij, S. V., Fuller, C., Steele, S. L., Veinotte, C. J., Razaghi, B., Robitaille, J. M., et al. (2018). Optimized knock-in of point mutations in zebrafish using CRISPR/Cas9. Nucleic Acids Res. 46, 9252. doi: 10.1093/nar/gky674

Qi, L. S., Larson, M. H., Gilbert, L. A., Doudna, J. A., Weissman, J. S., Arkin, A. P., et al. (2013). Repurposing CRISPR as an RNA-guided platform for sequencespecific control of gene expression. Cell 152, 1173-1183. doi: 10.1016/j.cell.2013. 02.022

Quach, H. N., Tao, S., Vrljicak, P., Joshi, A., Ruan, H., Sukumaran, R., et al. (2015). A Multifunctional mutagenesis system for analysis of gene function in zebrafish. G3 (Bethesda) 5, 1283-1299. doi: 10.1534/g3.114.015842

Raj, B., Wagner, D. E., Mckenna, A., Pandey, S., Klein, A. M., Shendure, J., et al. (2018). Simultaneous single-cell profiling of lineages and cell types in the vertebrate brain. Nat. Biotechnol. 36, 442-450. doi: 10.1038/nbt.4103

Ran, F. A., Cong, L., Yan, W. X., Scott, D. A., Gootenberg, J. S., Kriz, A. J., et al. (2015). In vivo genome editing using Staphylococcus aureus Cas9. Nature 520, 186-191. doi: 10.1038/nature14299

Rees, H. A., Komor, A. C., Yeh, W. H., Caetano-Lopes, J., Warman, M., Edge, A. S. B., et al. (2017). Improving the DNA specificity and applicability of base editing through protein engineering and protein delivery. Nat. Commun. 8:15790. doi: $10.1038 /$ ncomms15790

Regev, A., Teichmann, S. A., Lander, E. S., Amit, I., Benoist, C., Birney, E., et al. (2017). The human cell atlas. Elife 6:e27041. doi: 10.7554/eLife.27041

Schmidt, S. T., Zimmerman, S. M., Wang, J., Kim, S. K., and Quake, S. R. (2017). Quantitative analysis of synthetic cell lineage tracing using nuclease barcoding. ACS Synth. Biol. 6, 936-942. doi: 10.1021/acssynbio.6b00309

Seiler, C., Gebhart, N., Zhang, Y., Shinton, S. A., Li, Y. S., Ross, N. L., et al. (2015). Mutagenesis screen identifies agtpbp1 and eps15L1 as essential for T lymphocyte development in zebrafish. PLoS One 10:e0131908. doi: 10.1371/ journal.pone. 0131908

Shah, A. N., Davey, C. F., Whitebirch, A. C., Miller, A. C., and Moens, C. B. (2015). Rapid reverse genetic screening using CRISPR in zebrafish. Nat. Methods 12, 535-540. doi: 10.1038/nmeth.3360

Shen, M. W., Arbab, M., Hsu, J. Y., Worstell, D., Culbertson, S. J., Krabbe, O., et al. (2018). Predictable and precise template-free CRISPR editing of pathogenic variants. Nature 563, 646-651. doi: 10.1038/s41586-018-0686-x

Shmakov, S., Smargon, A., Scott, D., Cox, D., Pyzocha, N., Yan, W., et al. (2017). Diversity and evolution of class 2 CRISPR-Cas systems. Nat. Rev. Microbiol. 15:169. doi: $10.1038 /$ nrmicro.2016.184 
Sood, R., Carrington, B., Bishop, K., Jones, M., Rissone, A., Candotti, F., et al. (2013). Efficient methods for targeted mutagenesis in zebrafish using zinc-finger nucleases: data from targeting of nine genes using CompoZr or CoDA ZFNs. PLoS One 8:e57239. doi: 10.1371/journal.pone.0057239

Spanjaard, B., Hu, B., Mitic, N., Olivares-Chauvet, P., Janjuha, S., Ninov, N., et al. (2018). Simultaneous lineage tracing and cell-type identification using CRISPRCas9-induced genetic scars. Nat. Biotechnol. 36, 469-473. doi: 10.1038/nbt. 4124

Stemmer, M., Thumberger, T., Del Sol, Keyer, M., Wittbrodt, J., and Mateo, J. L. (2017). Correction: CCTop: an intuitive, flexible and reliable CRISPR/Cas9 target prediction tool. PLoS One 12:e0176619. doi: 10.1371/journal.pone. 0124633

Tanaka, S., Yoshioka, S., Nishida, K., Hosokawa, H., Kakizuka, A., and Maegawa, S. (2018). In vivo targeted single-nucleotide editing in zebrafish. Sci. Rep. 8:11423. doi: 10.1038/s41598-018-29794-9

Tessadori, F., Roessler, H. I., Savelberg, S. M. C., Chocron, S., Kamel, S. M., Duran, K. J., et al. (2018). Effective CRISPR/Cas9-based nucleotide editing in zebrafish to model human genetic cardiovascular disorders. Dis. Model Mech. 11:dmm035469. doi: 10.1242/dmm.035469

Toth, E., Czene, B. C., Kulcsar, P. I., Krausz, S. L., Talas, A., Nyeste, A., et al. (2018). $\mathrm{Mb}$ - and FnCpf1 nucleases are active in mammalian cells: activities and PAM preferences of four wild-type Cpf1 nucleases and of their altered PAM specificity variants. Nucleic Acids Res. 46, 10272-10285. doi: 10.1093/nar/gky815

Tseng, W. C., Loeb, H. E., Pei, W., Tsai-Morris, C. H., Xu, L., Cluzeau, C. V., et al. (2018). Modeling niemann-pick disease type $\mathrm{C} 1$ in zebrafish: a robust platform for in vivo screening of candidate therapeutic compounds. Dis. Model Mech. 11:dmm034165. doi: 10.1242/dmm.034165

Tu, M., Lin, L., Cheng, Y., He, X., Sun, H., Xie, H., et al. (2017). A 'new lease of life': FnCpfl possesses DNA cleavage activity for genome editing in human cells. Nucleic Acids Res. 45, 11295-11304. doi: 10.1093/nar/gkx783

Unal Eroglu, A., Mulligan, T. S., Zhang, L., White, D. T., Sengupta, S., Nie, C., et al. (2018). Multiplexed CRISPR/Cas9 targeting of genes implicated in retinal regeneration and degeneration. Front. Cell. Dev. Biol. 6:88. doi: 10.3389/fcell. 2018.00088

Varshney, G. K., and Burgess, S. M. (2014). Mutagenesis and phenotyping resources in zebrafish for studying development and human disease. Brief Funct. Genomics 13, 82-94. doi: 10.1093/bfgp/elt042

Varshney, G. K., and Burgess, S. M. (2016). DNA-guided genome editing using structure-guided endonucleases. Genome Biol. 17:187. doi: 10.1186/s13059016-1055-4

Varshney, G. K., Carrington, B., Pei, W., Bishop, K., Chen, Z., Fan, C., et al. (2016a). A high-throughput functional genomics workflow based on CRISPR/Cas9mediated targeted mutagenesis in zebrafish. Nat. Protoc. 11, 2357-2375. doi: 10.1038/nprot.2016.141

Varshney, G. K., Zhang, S., Pei, W., Adomako-Ankomah, A., Fohtung, J., Schaffer, K., et al. (2016b). CRISPRz: a database of zebrafish validated sgRNAs. Nucleic Acids Res. 44, D822-D826. doi: 10.1093/nar/gkv998

Varshney, G. K., Lu, J., Gildea, D. E., Huang, H., Pei, W., Yang, Z., et al. (2013). A large-scale zebrafish gene knockout resource for the genome-wide study of gene function. Genome Res. 23, 727-735. doi: 10.1101/gr.151464.112

Varshney, G. K., Pei, W., Lafave, M. C., Idol, J., Xu, L., Gallardo, V., et al. (2015a). High-throughput gene targeting and phenotyping in zebrafish using CRISPR/Cas9. Genome Res. 25, 1030-1042. doi: 10.1101/gr.186379.114

Varshney, G. K., Sood, R., and Burgess, S. M. (2015b). Understanding and editing the zebrafish genome. Adv. Genet. 92, 1-52. doi: 10.1016/bs.adgen.2015.09.002
Vrljicak, P., Tao, S., Varshney, G. K., Quach, H. N., Joshi, A., Lafave, M. C., et al. (2016). Genome-wide analysis of transposon and retroviral insertions reveals preferential integrations in regions of DNA flexibility. G3 (Bethesda) 6, 805-817. doi: $10.1534 / \mathrm{g} 3.115 .026849$

Watkins-Chow, D. E., Varshney, G. K., Garrett, L. J., Chen, Z., Jimenez, E. A., Rivas, C., et al. (2017). Highly efficient Cpf1-mediated gene targeting in mice following high concentration pronuclear injection. G3 (Bethesda) 7, 719-722. doi: 10.1534/g3.116.038091

Wu, R. S., Lam, I. I., Clay, H., Duong, D. N., Deo, R. C., Coughlin, S. R., et al. (2018). A rapid method for directed gene knockout for screening in G0 zebrafish. Dev. Cell 46:e114. doi: 10.1016/j.devcel.2018.06.003

Xu, S., Cao, S., Zou, B., Yue, Y., Gu, C., Chen, X., et al. (2016). An alternative novel tool for DNA editing without target sequence limitation: the structure-guided nuclease. Genome Biol. 17:186. doi: 10.1186/s13059-0161038-5

Yamada, M., Watanabe, Y., Gootenberg, J. S., Hirano, H., Ran, F. A., Nakane, T., et al. (2017). Crystal structure of the minimal Cas9 from Campylobacter jejuni reveals the molecular diversity in the CRISPR-Cas9 systems. Mol. Cell 65:e1103. doi: 10.1016/j.molcel.2017.02.007

Yang, L., Zhang, X., Wang, L., Yin, S., Zhu, B., Xie, L., et al. (2018). Increasing targeting scope of adenosine base editors in mouse and rat embryos through fusion of TadA deaminase with Cas9 variants. Protein Cell 9, 814-819. doi: 10.1007/s13238-018-0568-x

Yin, L., Maddison, L. A., Li, M., Kara, N., Lafave, M. C., Varshney, G. K., et al. (2015). Multiplex conditional mutagenesis using transgenic expression of Cas9 and sgRNAs. Genetics 200, 431-441. doi: 10.1534/genetics.115. 176917

Zafra, M. P., Schatoff, E. M., Katti, A., Foronda, M., Breinig, M., Schweitzer, A. Y., et al. (2018). Optimized base editors enable efficient editing in cells, organoids and mice. Nat. Biotechnol. 36, 888-893. doi: 10.1038/nbt.4194

Zetsche, B., Gootenberg, J. S., Abudayyeh, O. O., Slaymaker, I. M., Makarova, K. S., Essletzbichler, P., et al. (2015). Cpf1 is a single RNA-guided endonuclease of a class 2 CRISPR-Cas system. Cell 163, 759-771. doi: 10.1016/j.cell.2015.09.038

Zetsche, B., Strecker, J., Abudayyeh, O. O., Gootenberg, J. S., Scott, D. A., and Zhang, F. (2017). A survey of genome editing activity for 16 Cpf1 orthologs. bioRxiv [Preprint]. doi: 10.1101/134015

Zhang, Y., Qin, W., Lu, X., Xu, J., Huang, H., Bai, H., et al. (2017). Programmable base editing of zebrafish genome using a modified CRISPR-Cas9 system. Nat. Commun. 8:118. doi: 10.1038/s41467-017-00175-6

Zhang, Y., Zhang, Z., and Ge, W. (2018). An efficient platform for generating somatic point mutations with germline transmission in the zebrafish by CRISPR/Cas9-mediated gene editing. J. Biol. Chem. 293, 6611-6622. doi: 10. 1074/jbc.RA117.001080

Conflict of Interest Statement: The authors declare that the research was conducted in the absence of any commercial or financial relationships that could be construed as a potential conflict of interest.

Copyright (C) 2019 Liu, Petree, Requena, Varshney and Varshney. This is an openaccess article distributed under the terms of the Creative Commons Attribution License (CC BY). The use, distribution or reproduction in other forums is permitted, provided the original author(s) and the copyright owner(s) are credited and that the original publication in this journal is cited, in accordance with accepted academic practice. No use, distribution or reproduction is permitted which does not comply with these terms. 